Revue d'histoire de l'Amérique française

REVUE D.HISTOIRE DE L'AMÉRIQUE FRANÇAISE

\title{
La Rochelle et le Canada au XVIIe siècle
}

\section{Delafosse}

Volume 4, numéro 4, mars 1951

URI : https://id.erudit.org/iderudit/801666ar

DOI : https://doi.org/10.7202/801666ar

Aller au sommaire du numéro

Éditeur(s)

Institut d'histoire de l'Amérique française

ISSN

0035-2357 (imprimé)

1492-1383 (numérique)

Découvrir la revue

Citer cet article

Delafosse, M. (1951). La Rochelle et le Canada au XVIIe siècle. Revue d'histoire de l'Amérique française, 4(4), 469-511. https://doi.org/10.7202/801666ar d'utilisation que vous pouvez consulter en ligne.

https://apropos.erudit.org/fr/usagers/politique-dutilisation/ 


\title{
LA ROCHELLE ET LE CANADA
}

\author{
AU XVIIe SIECLE
}

Dans l'histoire du Canada français "l'épopée mystique" et l'organisation administrative et militaire qu'on ne saurait négliger sans mutiler gravement le passé canadien ne doivent pourtant pas faire oublier le jeu des intérêts matériels. Cette affirmation devrait paraître banale et cependant il faut bien constater que l'aspect économique des relations franco-canadiennes n'a pas encore assez retenu l'attention des chercheurs pour être bien éclairé. C'est pourquoi j'essaierai de préciser le rôle d'un des grands ports français des fourrures et de mettre en lumière les efforts des marchands rochelais pour entretenir des relations régulières avec la Nouvelle-France, la ravitailler en marchandises et en hommes et en retirer en retour les précieux castors. Je m'occuperai seulement des années 1630-1692, choix qui serait tout à fait arbitraire s'il ne m'était conseillé par la considération des travaux, soit achevés, soit en cours, étudiant le même sujet pour les périodes antérieure et postérieure ${ }^{1}$.

La liste des navires ayant fait le voyage La Rochelle-NouvelleFrance, qu'on trouvera en annexe, en même temps qu'elle donnera des précisions de détail souvent demandées, apportera les éléments d'une statistique du trafic. On souhaiterait en effet connaître le nombre des navires ayant quitté chaque année nos ports pour le Canada, on voudrait pouvoir présenter des chiffres cohérents et utilisables à côté d'affirmations générales. Est-ce possible? La liste ci-jointe est celle qu'ont permis de dresser les archives rochelaises,

1. Pour la période antérieure: Et. Trocmé, "Le commerce de La Rochelle à la fin du XVI ${ }^{\circ}$ s. et au début du XVII ${ }^{\circ}$ s." (encore inédit). Pour la période posterieure: These de doctorat de M. Robert en préparation sur les trafics coloniaux du port de La Rochelle au XVIII ${ }^{\circ}$ s.; M. Laveau se propose de consacrer un travail de même importance aux relations des ports français et du Canada à la même époque - Pour les recherches sur l'émigration, voir note 40. 
minutes notariales et fonds de l'amirauté. Je ne reviendrai pas longuement sur la critique de ces documents qu'on trouvera dans une étude analogue sur les Antilles'. Disons seulement que l'Amirauté paraît avoir conservé, à partir de 1670, par le dépôt des rôles d'équipage, l'indication du plus grand nombre de départs de navires. Quant aux minutes notariales, elles fournissent des renseignements dont on est certain qu'ils sont très incomplets sans qu'on puisse fixer le nombre des manquants; heureusement les années pauvres en documents de l'amirauté, $1630-1670$, semblent en revanche particulièrement riches en documents notariaux. En somme il faut pour La Rochelle renoncer à établir une liste absolument complète mais considérer que nous sommes pour les premières années en face de minima, et pour la période 1670, devant des chiffres susceptibles de légères modifications, mais solidement fondés.

On arrive ainsi pour $1632-1670$ à un total de 143 navires, soit une moyenne annuelle de 4 environ et pour les 24 années suivantes, de documentation plus sûre, à un total de 144 avec une moyenne de 6 , peu différente de la précédente.

Le trafic offre une continuité remarquable: ce n'est que pour une seule année, 1652, qu'il n'a pas été possible de relever de départs et les variations d'une année à l'autre et surtout d'une période de deux ans à la suivante, ont très peu d'amplitude. Il y a des décades plus actives comme $1640-50$ ou 1682-1692, mais si on compare le trafic canadien a celui des Antilles, on constate que le premier est dépourvu de ces démarrages subits, de ces années de pointe caractérisant le secońd et qu'à partir de 1660 il reste loin derrière lui en importance.

Cette régularité dans le chiffre des départs n'implique nullement que le commerce de La Rochelle avec la Nouvelle-France a eu tou-

2. Revre d'histoire des colonies, XXXV (1949), 238-281. La documentation du présent article a été tirée des Archives départementales de la Charente-Maritime qui conservent les fonds des notaires rochelais à l'exception des minutes Moreau (a la Bibliothèque de La Rochelle) et le fonds de l'Amirauté. Les documents de ce dernier fonds seront désignés par leur cote, les actes notariés par le nom du notaire suivi de la date. Il faut noter que les notaires rochelais passaient leurs actes tantót sur des registres, tantôt sur des feuilles volantes, donc qu'une même date peut renvoyer à un registre ou à une liasse. Les principaux notaires ayant rédigé des actes relatifs au Canada sont Teuleron, Cherbonnier, Moreau, Juppin, Chesneau, Tongrelou, Rivière. Sauf nécessité, je ne redonnerai pas les références déjà indiquées dans la liste des navires. 
jours le même aspect. Au début les marchands rochelais agissent comme correspondants des premiers colonisateurs d'Acadie et du Canada; puis ensuite certains trafiquent pour leur propre compte. Il faut étudier avec toute la précision possible chacune de ces diverses tentatives.

Emmanuel Leborgne et Charles de Menou d'Aulnay

On a quelquefois lourdement chargé Leborgne en traçant du sieur d'Aulnay un portrait peut-être idéalisé. Les minutes notariales sont des documents en général sans passion. Voyons ce qu'ils nous donnent. Leborgne était un marchand catholique, originaire du nord de la France et établi à La Rochelle où il faisait en compagnie d'E. Dussault un commerce actif de grains et de vins avec la Normandie, la Flandre, l'Angleterre et même la Baltique. Ses affaires marchaient bien puisqu'en 1641 il achète, dans la banlieue rochelaise, la maison noble de Fétilly en même temps qu'il est propriétaire de plusieurs navires. Dès 1634 il prête à Charles de Menou 900 livres à la grosse aventure sur le Petit Saint Luc; le taux de 22 pour cent est le même que celui qui est demandé par un autre marchand rochelais et la somme est d'ailleurs remboursée sans difficulté à la fin de l'année ${ }^{3}$. En 1642 les deux hommes sont entièrement liés: après avoir emprunté environ 16,000 livres, Charles de Menou, pour lui et "les autres intéressés aux habitations de Port Royal, la Heve et Pentagouet", donne procuration générale à Leborgne "pour faire achat de marchandises vivres et victuailles... qu'il conviendra envoyer par chacun ou dans un ou plusieurs navires... freter tous les navires qui lui seront nécessaires... emprunter les sommes de deniers à la grosse aventure... vendre les pelleteries de retour... faire recherche de ceux qui se trouveraient avoir apporté en fraude des pelleteries en ce royaume". Le même jour il lui accorde pour les opérations ci-dessus un droit de trois pour cent sur les marchandises d'aller et de deux pour cent sur les retours4. C'est bien ce rôle que nous lui voyons jouer les années suivantes. Il envoie en Acadie plusieurs navires chargés, en-

3. Juppin, 1634, 1 et 10/4.

4. Teuleron, $1642,16 / 5$, conventions et obligations. 
gage des hommes et poursuit la confiscation de navires ayant trafiqué en fraude, devant l'amirauté de La Rochelle, tribunal où le sieur d'Aulnay expose aussi ses démêlés avec Latour ${ }^{5}$. En 1644 un premier arrêt de comptes fait ressortir la situation lourdement obérée de l'Acadien: pour des envois faits en 1642 et 1643, après déduction du montant des pelleteries, il reconnait devoir au Rochelais 52,707 livres à payer dans six mois. De plus, pour 1644, sa dette augmente de 28,000 livres environ, soit en tout 81,000 livres, somme énorme pour l'époque ${ }^{6}$. Les retours des années suivantes et aussi les confiscations purent alléger ces dettes; pourtant en mai 1649 les relations se sont gâtées, car Leborgne nomme un procureur pour recevoir d'Aulnay jusqu'à 30,000 livres et en cas de défaillance le faire saisir. En 1653 et 1654 pourtant c'est la veuve et les enfants de l'Acadien qui sont ravitaillés par Leborgne. On sait que finalement il devait remplacer d'Aulnay et nous le voyons en effet prendre les titres de "Gouverneur et Lieutenant Général en Acadie" .

En somme il n'est pas douteux que Leborgne ait effectivement fait des envois réguliers et importants en Acadie pour d'Aulnay qui s'est fié à lui et a reconnu ses dettes. A priori il est suspect de voir Leborgne apparaître dans un contrat à deux titres, par exemple, de constater que Leborgne, propriétaire de navire, donne à fret son navire à Leborgne, procureur d'Aulnay; le prix alors nous semble volontiers élevé, 20,000 livres contre 14,000 livres pour un navire de même tonnage frété la même année par la Compagnie de la Nouvelle-France. Il est vrai que les risques de guerre en Acadie peuvent expliquer la différence. En 1654 Leborgne (il le déclare lui-même) dissimule aux enfants d'Aulnay qu'il prend part à la cargaison du Chateau fort à eux destinée. Aucun texte ne permet cependant d'accuser avec précision le rochelais de malhonnêteté et nous allons voir que ce n'est pas seulement dans les comptes Menou-Leborgne que les retours sont inférieurs aux envois.

5. B 5656, 1644, 9/1, rapport du maître de la Vierge; $i d ., 1645,8 / 11$ rapport du capitaine du Cardinal - Pour les confiscations: B 5656, 1645, 2/9,4/9,6/9, 4/11, $7 / 11 ; 1646,10 / 2$.

6. Teuleron, $1644,14 / 7$.

7. Teuleron, $1649,15 / 5 ; 1658,17 / 1$ et 19/2; Moreau, 1658, 14/1 B 5662, 1656, 6/3. 


\section{Charles de Saint Etienne et La Rochelle}

C'est aussi de La Rochelle que le grand rival d'Aulnay tira des marchandises et même des hommes ${ }^{8}$. Il y est à la fin de 1632 et au début de 1633: il fait enregistrer à l'amirauté une plainte de son lieutenant au fort Sainte Marie contre Guillaume Alexandre ${ }^{9}$, y règle les salaires des mariniers du Petit Saint Jean et de la Saubadine, puis affrète le Cheval Blanc et le Renard Noir sur lequel il s'embarque en avril. Samuel Georges et David Lomeron, deux notables marchands rochelais (notons qu'ils sont protestants) gèrent alors ses affaires, déclarant à l'amirauté les passagers qu'il emmène, $y$ faisant enregistrer ses concessions, empruntant pour lui de l'argent à la grosse aventure, affrétant des navires et lui envoyant des marchandises dont l'état de cargaison du Saint Jean en 1635 nous donne un long détail ${ }^{10}$. A la fin de l'année l'accord paraî́t rompu: selon les dires du rochelais, Charles de Saint Etienne a refusé, malgré ses engagements, de délivrer toutes les pelleteries à David Lomeron qui avait fait le voyage d'Acadie et à qui il a au contraire extorqué la promesse d'en remettre une partie au capitaine Lebert" ${ }^{11}$. En 1636 cepenidant Georges et Lomeron louent encore un navire pour Latour. Mais de cette même année à 1643 , c'est un autre "intendant" que eelui-ci a à La Rochelle: Guillaume Desjardins, sieur de Saint Val qui portait déjà ce titre en 1633 , lors de son voyage en Acadie, et qui, en 1636, est en même temps "Contrôleur provincial des guerres" à La Rochelle. Il remplit alors les fonctions assurées par les deux protestants rochelais; relevons en particulier les levées d'hommes, surtout de soldats qu'il fait en 1642 et $1643^{12}$. Le sieur de Latour est rencontré de nouveau à La Rochelle en 1650. Mais cette fois le lieutenant général en Acadie, "grand sagamos des Ouriquois" est détenu "es prisons royalles", d'ailleurs pour peu de temps puis-

8. Ce paragraphe est à compléter par: Abbé Couillard-Després "Aux sources de l'histoire de l'Acadie", Memoires de la Societe royale du Canada, XXVII (1933). Ce travail cite plusieurs textes rochelais que je n'ai pas repris.

9. B 5654, $1632,1 / 11$ et $1633,15 / 3$.

10. B $5654,1633,21 / 4 ; 1635,12 / 1,21 / 3$.

11. B $5654,1635,21 / 10$.

12. Cherbonnier, $1642,25-31 / 3$ et $1643,6 / 4$. 
qu'il demande son élargissement. C'est la dernière fois que nous le voyons dans notre port ${ }^{13}$.

\section{Les Tuffet et Cap Breton}

Jean Tuffet, un des associés de la Compagnie de la NouvelleFrance, était un marchand indiqué comme originaire de Bordeaux mais fixé à La Rochelle à l'époque qui nous occupe. Charles de Menou revenant sans fonds de l'Acadie à la fin de 1632 avait été heureux de l'y trouver pour l'aider à payer les équipages du Saint Jean et de l'Espérance. En 1637 et 1638 Jean Tuffet affrète des navires au nom de la Compagnie de Miscou et de celle de la Nouvelle-France. Mais sont surtout intéressantes ses relations et celles de son fils avec Pierre Desportes. L'île de Cap Breton avait été concédée à ce dernier en 1636 par la Compagnie de la Nouvelle-France. Une transaction de 1642, confirmée par les autres actes, expose bien les rapports de Desportes et de ses procureurs rochelais ${ }^{14}$. Il avait préposé, dès l'obtention de sa concession, Jean Tuffet à la "direction des envois et retours" et en effet à partir de cette date nous voyons Tuffet affréter chaque année un ou deux navires et engager des hommes ${ }^{15}$. Il était même devenu un véritable associé puisqu'il s'intéressait par moitié pour la troque, cependant que Desportes s'intéressait pour moitié dans la pêche; il est à remarquer en effet que les chartes-parties des navires allant à Cap-Breton prévoient, plus réguljèrement encore que les autres, à la fois la pêche et le port des marchandises. Mais la brouille apparaît bientôt. Les retours sont moindres que les envois et pourtant Desportes ne cesse d'envoyer de l'argent. Là-dessus Jean Tuffet meurt; son fils André, bien que sans connaissance des affaires (il est avocat), est entraîné à achever l'avitaillement en cours d'un vaisseau, puis à continuer l'année suivante. Desportes qui avait laissé passer des mois sans donner signe de rie envoie soudain comme procureur A. Cheffault qui

13. B $197 \mathrm{f}^{\circ} 104,1650,14 / 11$.

14. Teuleron, $1642,30 / 12$.

15. Engagements dans Teuleron, 1646, 5 et $10 / 4 ; 1637,27$ et $30 / 3 ; 1638,10,17$ et $20 / 3 ; 1640,14 / 3,29 / 4 ; 1641,1 / 4 ; 1643,11 / 2,2 / 3 ; 1644,8 / 1,15$ et $26 / 2,30 / 3$. 
réclame des comptes et de "grosses sommes de deniers". Mais après examen le rochelais apparaît comme créancier; il se fait attribuer les retours de l'année et concéder l'île en bail pour trois ans. Son frère ou neveu Louis Tuffet est établi Gouverneur à Saint-Pierre et en 1643 et 1644 les Tuffet expédient un navire par an pour leur compte. La ferme devait expirer le 1er janvier 1646; Desportes cède à partir de cette date Cap Breton à Me Gilles Guignard, sieur de la Fontaine, garde ordinaire provincial de l'artillerie en Poitou, Saintonge, Aunis. Tuffet préfère alors renoncer, en mai 1645, aux six mois de bail qui lui restent, car il sait qu'au premier janvier suivant il n'aura pas de navire à Cap Breton pour repasser hommes et effets et Guignard peut expédier dès cette année 1645 son navire l'Étoile. Les difficultés commencent dès le retour du bâtiment, en octobre, l'équipage se plaignant de n'être pas payé et refusant même de délivrer à Tuffet les pelleteries rapportées pour lui par le navire ${ }^{16}$. Les Tuffet paraissent avoir abandonné ensuite le commerce maritime pour se consacrer aux carrières judiciaires.

\section{Nicolas Denys}

De Nicolas Denys je dirai peu de choses car une étude récente a cité à peu près tous les textes connus à son sujet ${ }^{17}$. Rappelons seulement qu'il réside à La Rochelle de 1636 à 1650, "faisant les affaires" des Razilly, de la Compagnie de la Nouvelle-France, et même incidemment celles de Latour. Le financement des affaires qu'il mène pour son compte à partir de 1645 peut nous retenir plus longuement, comme plus ignoré. Il est alors propriétaire du Saint Joseph, navire de 300 tonneaux qu'il emploie tant à la pêche qu'à l'envoi de marchandises en Acadie; 1 parisien et 17 rochelais dont 16 marchands et un receveur des aides lui avancent à la grosse aventure les fonds nécessaires à l'armement. En temps prévu il est dans l'impossibilité de les rembourser si bien qu'il doit demander leur consentement quand l'année suivante il vient faire faire à son bateau un second voyage. Les retours de cette deuxième expédition lui permettent

16. B 5656, 1645, 9/10. Teuleron, $1645,5 / 5$.

17. R. Le Blant, "Les études historiques sur la colonie française d'Acadie", Revue d'histoire des Colonies, XXXV (1948): 94-102. 
de rendre le principal de sa dette, soit plus de 25,000 livres en vendant à ses créanciers 912 peaux d'originaux et 1,500 quintaux de poisson sec; mais les intérêts à raison de $25 \%$, soit plus de 6,000 livres, restent dus ${ }^{18}$. Parmi les prêteurs on trouve les principaux marchands rochelais, presque tous protestants, les Tessereau, Pagez, Bardet, Allaire, Vlamin, Van Sevenhoven, etc... Dans les années suivantes il va être de nouveau largement fait appel au capital rochelais, cette fois par la communauté de Québec.

\section{Les créanciers de la Communauté de Québec}

C'est à La Rochelle que se firent de nombreux armements et engagements pour la Compagnie de la Nouvelle-France et la Communauté des habitants de Québec. Après Tuffet nous voyons à l'œuvre, à partir de 1641, Antoine Cheffault, l'un des directeurs, puis Pierre Le Gardeur, sieur de Repentigny "directeur des embarquements" pour les habitants de la Nouvelle-France, et Noël Juchereau, qui prend le même titre et entre en conflit avec Olivier Le Tardif, commis de la Compagnie ${ }^{19}$. Mais, soit lacunes de la documentation, soit réalité des faits, nous ne constatons pas qu'ils font des emprunts importants, jusqu'à ceux des années 1648-1651 qui nous sont révélés, ainsi que les démêlés qui s'en suivent, par de nombreux textes.

En juillet 1648 Noël Juchereau, procureur des habitants de la Nouvelle-France, souscrit une obligation simple de 21,800 livres à un banquier Auger Duchanin; de plus il avait divers autres créanciers, ceux-là à la grosse aventure, qui, à la fin de l'année, font saisir des castors vendus à Jacques Pepin ${ }^{20}$. Au début de 1649 Jean-Paul Godefroy et Jean Juchereau sont de nouveau à La Rochelle; deux autres banquiers protestants leur prêtent à la grosse aventure, à 25 pour cent, l'un Henry Bardet 20,000 livres, l'autre, Samuel Pagez, 10,000; une dizaine d'autres marchands rochelais donnent ensemble aux mêmes conditions, 15,000 livres. En décembre même scénario

18. Teuleron, $1646,9 / 3$.

19. Teuleron, $1642,6 / 4-16 / 5 ; 1643,28 / 3,30 / 3,1 / 4-14 / 4,20 / 5 ; 1644,18 / 4-5 / 5$; $1645,10[6,18 / 12 ; 1647,2 / 1,5 / 5 ; 1648,22 / 4$. B 5656, 1645, 20/5. B 5657, 1646, 18/7. La charte-partie passée par Le Tardif devant Moreau, 1646, 22/8 prévoit l'arrét possible du navire par les habitants. Les textes rochelais peuvent ainsi apporter quelques lueurs sur les démêlés des Canadiens entre eux.

20. Michelon, 1648, 1/7. Cherbonnier, 1648, 10/12. 
que l'année précédente: ayant su que, contrairement aux engagements pris, les navires canadiens et notamment le Grand Cardinal sont sur le point de faire retour ailleurs qu'à La Rochelle, une partie des créanciers donne pouvoir de faire saisir les cargaisons jusqu'au montant des créances ${ }^{21}$. En 1650 pourtant de nouvelles obligations furent souscrites mais ne purent pas non plus être remboursées dans l'année et c'est en avril 1651 qu'une vente de plus de 10,000 livres pesant de castors faite pour 65,000 livres tournois à Robert Poquelin de Paris, permet de répartir entre les créanciers 62,300 livres sur 90,785 dues $^{22}$. Sur ces sommes il n'est pas possible de faire la part exacte des vingt-deux rochelais qui approuvent la transaction; mais ce paiement eut pour effet de rétablir la confiance pour un nouvel appel de fonds qui ne tarde pas: Jean Bourdon, agissant en vertu d'ordres de la part du Conseil pour les affaires de la NouvelleFrance $^{23}$ et comme procureur de la Communauté de Québec, passe, entre le 15 mai et le 15 juillet, de nombreux contrats d'emprunt à La Rochelle: il ne nous en est resté que 28 rédigés par le notaire Teuleron pour une somme globale d'environ 50,000 livres sur les navires le Saint Joseph et la Vierge, mais nous savons que d'autres obligations rédigées par le notaire Peronneau concernaient le navire le Passemoy et qu'en tout les créanciers rochelais étaient au moins 47, comptant parmi eux petits et gros marchands, catholiques et protestants. Ils n'eurent pas à se féliciter de cette affaire et, bien loin de toucher les $30 \%$ de profit qui avaient été stipulés, ils ne rentrèrent même pas dans leur capital. En effet sur les quatre navires revenant de Canada à la fin de 1651, l'un, la Vierge, ne put aller plus loin que les Açores et resta à la Tercière hors d'état de continuer sa route; un autre le Saint Joseph, fut confisqué à son arrivée à La Rochelle par le gouverneur rebelle du Dognon; enfin les deux derniers, le Passemoy et le Petit Saint Jean arrivèrent au port, mais avec de bien maigres cargaisons, puisque les six poinsons de castors du Passemoy ne produisirent que 10,326 livres presque complètement absorbées par le fret du navire ${ }^{24}$. On ignore si les rochelais arrivèrent à faire rapporter des Açores la cargaison de la Vierge. L'année

21. Michelon, 1649, 22/3. Teuleron, $1649,29 / 5$. Cherbonnier, 1649, 15/12.

22. Teuleron, $1651,5 / 5$ et $13 / 6$.

23. Ces "ordres" datés du 29/10/1650 sont déposés dans la liasse Teuleron. Ils mériteraient une publication complète ainsi que ceux du 15/11/1651. 
suivante il n'était certes pas question de faire un emprunt aux Rochelais, préoccupés seulement d'essayer de récupérer un peu de leur argent. Ils sont alors en conflit avec deux d'entre eux, Bérandin et Pagez, qui se sont portés dès 1650 acheteurs de tous les castors de la communauté. Nous trouvons cependant comme procureur de la communauté, en vertu d'instructions du Conseil de Québec, le rochelais Jean Garos. En 1655, après des débats restés obscurs, on en vient à une transaction. Une sentence arbitrale rendue à Rouen le 20 septembre est acceptée par les parties, devant la Cour consulaire de cette ville. Les $132,359 £ 1.17 \mathrm{~s}$. empruntés sur la Vierge et le Saint Joseph en 1651 (il s'agit vraisemblablement du total des obligations et non pas seulement de celles passées à La Rochelle) sont réduites à 81,480 livres, l'obligation de 1,000 livres tombant ainsi à 615, payables en deux termes, fin 1657 et fin 1658 . Ces clauses ne furent pas respectées, puisqu'en 1673 les créanciers rochelais de la Communauté de Québec nomment des procureurs pour la perception du droit de 10 pour cent entrant et sortant de la NouvelleFrance ${ }^{25}$. Ainsi la tentative de la Communauté de Québec pour assurer elle-même son ravitaillement ce terme étant entendu dans son sens large, s'était soldée par un échec: les pelleteries de retour n'avaient pas suffi à payer les marchandises sur lesquelles, il est vrai, les fournisseurs rochelais prétendaient faire de copieux bénéfices. Par suite de cette défaillance des Canadiens, la voie du commerce libre s'ouvrait devant les marchands rochelais. Quelquesuns allaient tenter leur chance.

\section{LES MARCHANDS ROCHELAIS}

Dès 1650 nous avons mention d'une permission à un navire rochelais, le St Jean, pour le voyage de Québec; les instructions données à la fin de 1651 par le Conseil de Québec prévoient l'octroi aux sieurs Bérandin et Pagez, pour les récompenser de leurs bons offices, de la permission d'envoyer à Québec des marchandises pour leur compte particulier. Enfin l'Amirauté de La Rochelle nous a conservé un "conge" donné en 1655 au sieur Peron pour envoyer ou faire venir dans le St Laurent un navire chargé de vivres et marchandises, avec

24. Teuleron, $1652,9 / 1,12 / 1,2 / 2,6 / 3 ; 1653,14 / 1 ;$ B 5661, 1655, 23/3.

25. Moreau, $1655,7 / 12$. Teuleron, $1673,10 / 7$. 
défense de faire lui-même la traite des pelleteries et obligation d'amener un homme de travail par 16 tonneaux de jauge ${ }^{26}$. On ne peut suivre exactement dans les détails ce régime des congés, mais la liste des départs montre qu'à partir de 1650 les marchands rochelais ont eu la possibilité d'envoyer leurs navires au Canada. Leur nombre reste assez restreint et, dans les années $1655-1692$, je ne compte guère que six ou sept maisons ayant armé avec quelque constance pour la Nouvelle-France. Je laisse bien entendu de cấté pour l'instant les petits marchands faisant seulement envoi de quelques ballots de marchandises.

J'ai cité plus haut François Peron qui reçoit un congé en 1655. Jusqu'en 1663 il envoie annuellement à Québec un navire qui est souvent son propre navire, le Taureau, mais en 1664 il fait faillite, à la suite d'un essai malheureux de commerce aux Antilles $^{27}$. De même Jacques Pépin, autre marchand protestant et d'ailleurs un des pionniers du commerce antillais, arme plusieurs navires pour Québec de 1656 à 1662; notons aussi de sa part une levée d'une vingtaine d'hommes en $1656^{28}$. Vers les mêmes années Jacques Mousnier, marchand catholique, fait aussi quelques armements et quelques engagements; Alexandre Petit de 1666 à 1673, se débat péniblement au milieu d'affaires embarrassées, au terme desquelles on voit ses créanciers, les grands marchands protestants Bion, Depont, Stevenot, etc... saisir ses castors ${ }^{29}$. Jean Gitton, marchand catholique, commence par une association avec Peron, en 1656, un trafic canadien qui sera de longue durée et qu'il mènera en compagnie de divers autres marchands dont le protestant François Duprat jusqu'en 1689 au moins; il fait plusieurs fois le voyage ${ }^{30}$.

26. B $5661,1655,2 / 10$.

27. Cherbonnier, 1656, 13/4: Association Peron-Gitton-Cherbonnier, 1657, 16 et 24/3, 10 et 13/4, 17/5, 9/8; Emprunts et accords relatifs au voyage de 1657 . Cherbonnier, 1655, 29/6-2/8; 1656, 11/4; 1657, 10/4; 1658, 14/5; 1659, 27/6: engagements au nombre de 56 - La charte-partie Cherbonnier, 1661, 3/6 prévoit le passage de 60 a 70 passagers pour le compte de Pierre Fillye, commis de marchands de Rouen.

28. Moreau, 1656, 23/3-5/4.

29. Drouyneau, $1673,1 / 3$.

30. La liste des navires indique de nombreux armements de Jean Gitton et compagnie. B 5676 no 132 donne avec l'état de cargue de l'Aigle noir en 1677 un exemple de cargaison pour Québec: 33 muids de sel, 60 barriques de farine, 50 barriques d'eau-de-vie, 6 tonneaux de vinaigre, 250 balles de draperies et toile - Autre exemple dans B 5672 no 41. 
Parmi ces marchands rochelais en relations avec le Canada il faut faire une place à part, pour l'importance, à la compagnie Gaigneur-Grignon, composée d'Antoine Grignon, de son fils Jean et de ses gendres Pierre Gaigneur et Arnaud Perez, tous catholiques. Pierre Gaigneur appartenait à une vieille famille rochelaise et son père, Louis Gaigneur, avait déjà commandé pour Tuffet à Cap Breton et armé en 1650 un navire pour Québec. De leur côté, dès 1651 et 1654, A. Grignon et A. Perez sont en rapport avec les Jésuites de Québec et le Canadien Jacques Maheut ${ }^{31}$. A partir de 1656 l'association Gaigneur-Grignon paraît formée, Gaigneur y occupant la première place jusqu'en 1673 pour s'effacer ensuite. La liste cijointe donne le détail de ses nombreux armements pour Québec, la plupart des navires étant loués, car la compagnie n'est propriétaire d'un bâtiment important, au nom symbolique de la Nouvelle-France, qu'à partir de 1667. Malheureusement ses opérations au Canada nous échappent et quelques ventes de castors ne suffisent pas à nous éclairer sur le volume de ses transactions. Mais ses difficultés financières se manifestent assez clairement quelquefois. Ainsi le second voyage de Gaigneur et Grignon, celui de 1657, paraît se terminer par un déficit; ils vendent en effet pour 16,491 livres 5 sols de castors; mais le fret du navire et les salaires de l'équipage absorbent aussitôt plus de 10,400 livres et ils ne peuvent payer que 6,050 livres à leurs créanciers, gros aventuriers à qui il est dâ 15,730 livres, soit 12,000 livres de principal plus les intérêts à 30 pour cent environ. Il faut que les créanciers où nous retrou vons les noms des riches marchands et banquiers qui prêtaient à Denys et à la Communauté de Québec, consentent de nouveaux prêts sur les marchandises à charger dans les navires qui seront expédiées à Québec en 1658 et finalement l'argent n'est totalement remboursé qu'au début de $1659^{32}$. Le remboursement des obligations passées en 1662, 1663, 1664 fut de même bien difficile; Gaigneur et Grignon donnent comme raison l'impossibilité où ils ont été d'écouler leurs marchandises à Québec: aussi les 21 créanciers qui, en 1662, leur avaient prêté 24,000 livres à 24 pour cent, doivent-ils leur accorder des délais, ce

31. Teuleron, 1651. 30/3; Moreau, 1654, 23/5 - En 1657, 33 engagements par Grigonon. Gaigneur et Massé dans Moreau, 3/2-13/3.

32. Moreau, $1658,3 / 4$ avec quittance de $1659,13 / 2$. 
que tous ne font pas de bon gré. En 1664, pour retrouver du crédit, Gaigneur et compagnie sont obligés de vendre d'avance leurs pelleteries encore à venir du Canada, les acheteurs versant immédiatement une partie du prix en obligation à la grosse aventure ${ }^{33}$. En 1666 et 1667 , années où Gaigneur arme de nombreux navires, ses affaires paraissent mieux aller, malgré une question embrouillée de saisie, puisqu'il achète un navire de $250 \mathrm{tx}$., une maison et contribue en même temps à la reconstruction de Saint Jean, son église paroissiale $^{34}$. Quelques années plus tard Gaigneur, qui a fait une tentative malheureuse de traite négrière, ne paraît plus guère. Antoine Grignon meurt, mais Jean Grignon et Arnaud Perez continuent à commercer avec le Canada. La famille Grignon paraît à ce moment avoir gagné une certaine aisance; et la fille de Jean, Louise Grignon, épouse Jacques Le Ber, de Montréal, qui faisait à La Rochelle les affaires de la Compagnie du Nord. ${ }^{35}$

En 1689 celui qui remplissait ces fonctions, était Jean Gitton dont j'ai parlé plus haut et qui était associé à François Duprat et François Pachot, "intéressés" comme lui dans la compagnie qu'ils représentaient. Un arrêté de compte relatif aux castors apportés dans le navire du Roi, le Soleil d'Afrique, porte sur 108,000 livres, ce qui paraît une très grosse somme ${ }^{36}$. D'autres actes antérieurs d'une dizaine d'années peuvent aussi nous donner un aperçu de l'importance du trafic des castors. Charles Aubert, sieur de Chesnaye, marchand de Québec, mais qui réside souvent à La Rochelle d'où partent ses navires et où il a un associé, cède en 1678 les $2 / 3$ de sa por-

33. Cherbonnier, $1662,20 / 5$ et Teuleron, même jour, avec quittances de 1663 et 1664. Moreau, 1663, 20/4; Teuleron, 1663, 3 et 23/4.

34. Parmi les engagements faits par Gaigneur, signalons la grande levée de 1665 où figure Mathurin Villeneuve: Teuleron, 23/3-18/4 - B 5669 no 29 donne pour 1670 la cargaison de retour de la Nouvelle France: 860 peaux d'orignaux, 10 ballots et 3 paquets de castors, du bois à brâler.

35. Teuleron, $1675,3 / 11$. Inventaire après décès d'Antoine Grignon qui indique quelques créances au Canada. Un deuxième fils, Auger Grignon devait y résider. Rivière; 1689, 25/4 - Contrat de mariage Grignon-Le Ber et 1693, 24/1 inventaire après décès de ce dernier.

36. Rivière, 1689, 22/4. La question des compagnies, depuis celles des premières années jusqu'à la compagnie du Nord de Canada, de pêche sédentaire d'Acadie, en passant par la Compagnie des Indes Occidentales, demanderait une étude non limitée à La Rochelle. 
tion "en la ferme et commerce de Canada et revente de castors" dont avec Bazire et Joulin il avait primitivement la moitié. Ces actes sont assez confus et demanderaient à être complétés; mais il en résulte au moins que cette cession est faite pour plus de 350,000 livres, le "fond capital" de la société étant fixé à 1,061,348 livres. Il en ressort également que la grande finance parisienne qui est aussi intéressée dans la compagnie du Nord de Canada, participe à ces affaires: Jean-Baptiste Delagny, Louis Carel, receveur général des finan'ces, Charles Patru, Gabriel Berthelot de Creil, etc., sont cités par ces contrats, ain’si que dans les acheteurs de "castors de chapellerie", la maison Pocquelin de Paris que nous avons déjà rencontrée comme acheteuse des castors de la Communauté de Québec ${ }^{37}$. Ajoutons enfin que les pelleteries ou au moins partie d'entre elles sont réexpédiées à Amsterdam et Hambourg, alimentant ainsi le trafic international. ${ }^{38}$

A côté de ces grands noms et pour avoir une vue complète du commerce canadien, il faut noter de nombreuses tentatives de petits marchands, d'artisans même, qui empruntent à la grosse aventure quelques centaines de livres, achètent quelques ballots de marchandises, les chargent sur les navires de négociants et vont tenter leur chance en Nouvelle-France. Souvent ils s'associent à deux ou trois, l'un faisant le voyage, l'autre restant à La Rochelle pour négocier les retours; parfois un Rochelais ou un Poitevin s'associe ainsi à un Canadien, tel ce marchand de la Châtaigneraie qui confie à Joseph Petit, sieur de Bruneau, et à Léonard Pitouin, marchands de Québec, des étoffes, à charge de les vendre à moitié de bénéfice ${ }^{30}$. Nous voyons ainsi passer à La Rochelle de nombreux Canadiens qui

37. Teuleron, $1678,23 / 4$.

38. Teuleron, 1679, 24/6 et 17/7: Etienne Joulin, associé de Ch. Aubert, envoie deux navires chargés de pelleteries et sel en Hollande - B 5671 no 156: déclaration d'A. Chapman qui depuis de nombreuses années fait le négoce des sirops et peaux d'orignaux de La Rochelle à Hambourg.

39. Drouyneau, 1679, 12/6 - Association Feriou-Compain: Teuleron, 1656, 4/2 et Cherbonnier, 1657, 28/2 - Association Ponty-Breschon: Cherbonnier, 1658, 2/5 Association François-Chambert: Moreau, 1658, 16/5. - Association BestreauPichon: Cherbonnier, 1657, 3 et 19/2, B 5664 no 69. Association Vieney-PachotAramy: Cherbonnier, 1657, 13/3 - Association A. Turpin de Québec - J.-B. Lebeau de Nantes: Juge, 1693, 8/5 - Concordat de Paul Defay et Simon Maes qui font une faillite de 30,000 livres: Juge, 1677, 15/5. 
viennent $y$ chercher des marchandises ou des hommes; beaucoup "d'engagés" ont en effet quitté par notre port l'ancienne France pour la nouvelle.

\section{Con CLusion}

Après avoir ainsi passé en revue les principaux marchands rochelais qui ont commercé avec la Nouvelle-France, on aperçoit aisément des traits communs à ces diverses entreprises. Lors de la première période qui va jusque vers 1650 , le Rochelais est correspondant d'un colonisateur acadien ou canadien; pendant des années il lui envoie des navires, des hommes et des marchandises que ne compensent pas les pelleteries adressées en retour; arrive un moment où les relations se gâtent. La différence des situations et des mentalités est évidente: certes l'idée de profit n'est pas étrangère au pionnier; mais, engagé dans les difficultés et les dangers, il n'a pas les yeux fixés sur les comptes que le marchand tient, sinon avec honnêteté du moins avec soin. Quand on fait le bilan, il apparaît négatif pour le colon et son créancier cherche alors à saisir des gages; Leborgne remplace d'Aulnay, Tuffet prend Cap Breton à bail, les créanciers de Québec essaient d'y lever des taxes.

La situation ne change pas fondamentalement quand certains marchands se mettent à envoyer en Canada des navires pour leur propre compte. Pour la plupart ce sont des catholiques qui ont besoin d'appui financier; ils ont recours aux gros marchands et ban-

40. J'ai peu insisté sur cette question des engagés qui doit être traitée de façon complète par $\mathbf{M}$. Debien pour le XVIIe siècle et $\mathbf{M}$. Gaucher pour le XVIIIe. De M. Debien, voir un premier travail provisoire: Les engagés partis de La Rochelle Parmi les Canadiens de passage à La Rochelle ou s'y embarquant: Pierre Leclerc, sieur de la Vigne (testament): Teuleron, 1644, 7/7. Léonard Leclerc, sieur de Narbonne (testament): Teuleron, 1645, 21/6 - Antoine Taboureau (testament, legs a des habitants de Québec): Teuleron, 1646, 25/2. - Augustin Hébert de Montréal: Teuleron, 1647, 2/5 - Jean Humeau de Québec: Teuleron, 1648, 13/5 Guillaume Vignal, prêtre (testament): Juppin, 1648, 13/5 - Thomas Clemanceau, sa femme et Pierre Biron: Juppin, 1648, 17/5 - Jean Chaillot. Moreau, 1654, 8/3. - Pierre Mainville: Moreau, 1656, 13/3 - Michel Peltier, des Trois Rivières et Médard Chouard, sieur des Groseilliers, "général de la flotte des autavois": Moreau, 1661, 6 et 27/5. - P. Boucher, sieur des Grosbois: Teuleron, 1662, 2/5 et Moreau, 1662, 17/5 - Jean Plante habitant la côte de Beaupré; Teuleron, 1665, 14/5. Louis Pinard, chirurgien aux Trois-Rivières: Cherbonnier, 1666, 17/5. - Denis Guion, marchand de Québec: Teuleron, 1670, 21/4 - Hervieux, propriétaire du fief du Vieil Logis ou Pipeguereche côte d'Acadie: Teuleron, 1680, 17/12. 
quiers protestants qui à La Rochelle détiennent les capitaux. Ceuxci les leur avancent dans les conditions habituelles à cette époque: prêts à la grosse aventure avec intérêt élevé qui ici atteint facilement 30 pour cent. Il semble bien que les remboursements soient difficiles. Sans doute il faut tenir compte de la documentation et songer qu'un règlement de compte embrouillé produit plus de paperasse qu'une entreprise sans complications. Nous sommes ainsi mieux renseignés sur les difficultés que sur les succès. Néanmoins, si, contrairement à ce qui se passe dans le négoce avec les Antilles, nous constatons peu de faillites retentissantes, l'impression reste que les armateurs trafiquant avec le Canada vivent assez péniblement et fréquemment leurs créanciers ont recours à la saisie des pelleteries, ce qui est en somme la répétition du scénario de la première période.

Quand les armateurs s'excusent de ne pouvoir rembourser, la raison donnée est l'insuffisance des retours qui explique le maintien du trafic au chiffre de quelques navires par an, tandis qu'au même moment le sucre assure au trafic antillais un volume bien supérieur. On ne se plaint pas que les castors ne se vendent pas, mais qu'il n'y en ait pas assez. Nous manquons complètement d'une statistique des pelleteries importées à La Rochelle. Ce que l'on voit, ou plutôt on soupçonne, ce sont les efforts d'une minorité pour s'assurer un monopole: ainsi deux riches marchands protestants, envers lesquels le Conseil se montre reconnaissant, se portent acquéreurs de tous les castors de la communauté de Québec; ainsi nous voyons les financiers parisiens s'intéresser à des ventes de pelleteries dont l'importance parait considérable par rapport au volume des affaires traitées par les petits armateurs.

Telles sont les réflexions que peut suggérer l'étude des relations de La Rochelle avec le Canada au XVIIe siècle. Bien entendu cette étude devrait être prolongée d'une part par les travaux sur l'époque antérieure, d'un caractère tout différent selon $M$. Trocmé, et sur l'époque postérieure qui, d'après M. Robert, apparaît comme plus semblable, d'autre part par des recherches sur les autres ports français. Ce n'est qu'après cela qu'on pourra juger si mes conclusions valent pour l'ensemble du trafic franco-canadien.

M. Delafosse, Archiviste de la Charente Maritime, France. 
Liste des navires ayant fait le voyage entre La Rochelle et le Canada de 1632 à 1693.

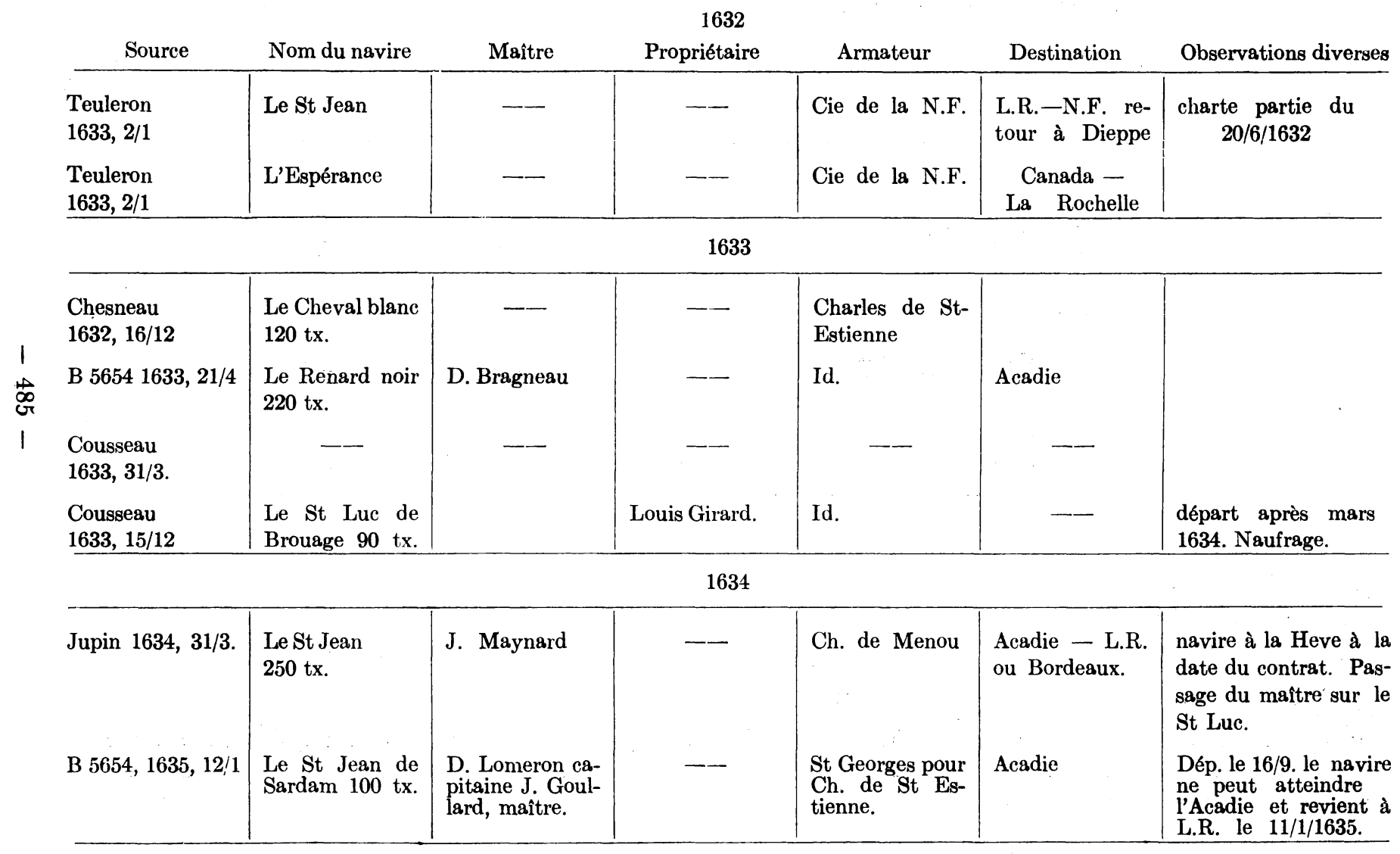


1635

\begin{tabular}{|c|c|c|c|c|c|c|}
\hline Source & Nom du navire & Maître & Propriétaire & Armateur & Destination & Observations diverses \\
\hline Moreau 1635, 6/4 & $\begin{array}{l}\text { Le Pigeon blanc } \\
200 \text { tx. }\end{array}$ & A. Camus. & $\begin{array}{l}\text { J. Corvé de St } \\
\text { Martin de Ré. }\end{array}$ & $\begin{array}{l}\text { S. Georges pour } \\
\text { Ch. de St Es- } \\
\text { tienne. }\end{array}$ & $\begin{array}{l}\text { Acadie, port de } \\
\text { La Tour. }\end{array}$ & $\begin{array}{l}\text { Pêche et port de mar- } \\
\text { chandises. Autre con- } \\
\text { trat du } 6 / 4 \text { perdu. }\end{array}$ \\
\hline $\begin{array}{l}\text { В } 5654 \\
1635,21 / 10 .\end{array}$ & $\begin{array}{l}\text { Le St Pierre de } \\
\text { Marennes. }\end{array}$ & - & - & Id. & Id. & $\begin{array}{l}\text { Dép. le 17/4, arrivee } \\
\text { le 5/6. Retour: dép. } \\
\text { le 29/9, arr. \& L.R. } \\
\text { le 20/10, avec le Pi- } \\
\text { geon blanc. }\end{array}$ \\
\hline
\end{tabular}

1636

\begin{tabular}{|c|c|c|c|c|c|c|}
\hline $\begin{array}{l}\text { Moreau } \\
1636,16 / 4\end{array}$ & $\begin{array}{l}\text { Le Croissant de } \\
\text { L.R. } 60 \text { tx. }\end{array}$ & J. Jamain & J. Jamain & $\begin{array}{l}\text { S. Georges pour } \\
\text { Ch. de St Es- } \\
\text { tienne. }\end{array}$ & $\begin{array}{l}\text { Acadie, le long } \\
\text { de la cốte. }\end{array}$ & \\
\hline $\begin{array}{l}\text { Teuleron } \\
1636,10 / 4\end{array}$ & $\begin{array}{l}\text { Le Don de Dieu } \\
\text { de Chaillevette. } \\
90 \mathrm{tx} .\end{array}$ & J. Vignaud & J. Derideau & $\begin{array}{l}\text { J. Taffet pour } \\
\text { P. Desportes. }\end{array}$ & $\begin{array}{l}\text { Cap Breton ha- } \\
\text { bitation ste An- } \\
\text { ne et pêche. }\end{array}$ & \\
\hline $\begin{array}{l}\text { B } 5654 \text { et Juppin } \\
1636,20 / 6 \text {. }\end{array}$ & $\begin{array}{l}\text { Le St Jean } 250 \\
\text { tx. }\end{array}$ & P. Sovic & - & N. Denys & & Retour en Septembre. \\
\hline $\begin{array}{l}\text { Teuleron } \\
1636,12 / 4\end{array}$ & $\begin{array}{l}\text { Le Nicolas de } \\
\text { St Gilles, } 100 \text { tx. }\end{array}$ & H. Langevin & - & $\begin{array}{l}\text { J. Tuffet pour la } \\
\text { Cie de la N.F. }\end{array}$ & $\begin{array}{l}\text { Miscou et } \\
\text { Tadoussac. }\end{array}$ & \\
\hline
\end{tabular}

1637

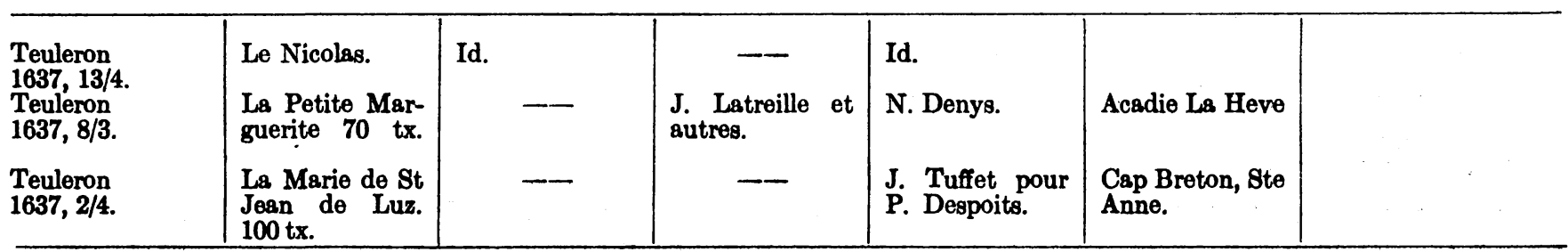




\begin{tabular}{|c|c|c|c|c|c|c|}
\hline $\begin{array}{l}\text { Teuleron } \\
1638,8 / 4\end{array}$ & Le Petit St Jean & J. Coupereau. & - & $\begin{array}{l}\text { J. Tuffet pour la } \\
\text { Cie de la N.F. }\end{array}$ & $\begin{array}{l}\text { Miscou \& } \mathrm{Ta}- \\
\text { doussac. }\end{array}$ & $\begin{array}{l}\text { Retour a la fin de } \\
\text { l'année. }\end{array}$ \\
\hline $\begin{array}{l}\text { Teuleron } \\
1638,23 / 4\end{array}$ & La Marie Mar- & N. Ecussard. & - & Id. & Tadoussac. & \\
\hline $\begin{array}{l}\text { Teuleron } \\
1638,28 / 3\end{array}$ & $\begin{array}{l}\text { vette. } \\
\text { Le Soleil de St } \\
\text { Gilles, } 140 \text { tx. }\end{array}$ & M. Duhalde & $\begin{array}{l}\text { L. Cadou \& au- } \\
\text { tres. }\end{array}$ & J. Tuffet. & $\begin{array}{l}\text { Cap Breton, Ste } \\
\text { Anne et pêche. }\end{array}$ & \\
\hline
\end{tabular}
Gilles, 140 tx. tres. Anne et pêche.

\begin{tabular}{|c|c|c|c|c|c|c|}
\hline \multicolumn{7}{|c|}{1639} \\
\hline $\begin{array}{l}\text { Teuleron } \\
1639,24 / 12\end{array}$ & $\begin{array}{l}\text { Le St Pierre, } \\
120 \text { tx. }\end{array}$ & - & - & - & $\begin{array}{l}\text { Revient du Cap } \\
\text { Breton et pêche }\end{array}$ & \\
\hline \multicolumn{7}{|c|}{1640} \\
\hline $\begin{array}{l}\text { Moreau } \\
1640,26 / 3\end{array}$ & $\begin{array}{l}\text { L'Amitié de La } \\
\text { Rochelle } 250 \\
\text { tx. }\end{array}$ & J. Jamain & $\begin{array}{l}\text { J. Pépin, } \\
\text { J. Jamain, } \\
\text { G. Desjardins }\end{array}$ & $\begin{array}{l}\text { G. Desjardin, de } \\
\text { St. Val pr. Ch. } \\
\text { de St Estienne. }\end{array}$ & $\begin{array}{l}\text { Acadie, ret. L.R. } \\
\text { ou Brouage }\end{array}$ & $\begin{array}{l}\text { Le navire est vendu } \\
\text { par ses propriétaires } \\
\text { le } 7 / 11 / 1641 \text {. }\end{array}$ \\
\hline $\begin{array}{l}\text { Moreau } \\
1640,21 / 9\end{array}$ & $\begin{array}{l}\text { Le Jacques de } \\
\text { Chaillevette } 70 \\
\text { tx. }\end{array}$ & J. Guyonneau. & J. Dherideau. & Id. & Id. & \\
\hline $\begin{array}{l}\text { B } 5656 \\
1646 \text {, 30/10 et } \\
\text { Teuleron } \\
1641,27 / 3 .\end{array}$ & $\begin{array}{l}\text { Le St François } \\
\text { de Pentagouet }\end{array}$ & J. Lebœuf & $\begin{array}{l}\text { Cie de la N.F. et } \\
\text { autres. }\end{array}$ & Ch. de Menou & Acadie & . \\
\hline $\begin{array}{l}\text { Teuleron } \\
1640,24 / 3 .\end{array}$ & Le St Jean & A. Camus. & - & - & - & $\begin{array}{l}\text { N. Denys fait passer } \\
25 \text { h. et } 5 \text { f. sur le nav } \\
\text { qui va faire la peche. }\end{array}$ \\
\hline $\begin{array}{l}\text { Moreau } \\
1640,21 / 3 . \\
\text { B 5656 }\end{array}$ & $\begin{array}{l}\text { Le Soleil } 130 \text { tx. } \\
\text { Le St Pierre, } \\
140 \text { tx. }\end{array}$ & $\begin{array}{l}\text { E. Camus. } \\
\text { M. Duhalde. }\end{array}$ & B. Pineau & $\begin{array}{l}\text { B. Pineau. } \\
\text { J. Tuffet } \\
\text { J. Tuffet. }\end{array}$ & $\begin{array}{l}\text { Cap Breton et } \\
\text { péche } \\
\text { Id. }\end{array}$ & - \\
\hline
\end{tabular}


1641

\begin{tabular}{|c|c|c|c|c|c|c|}
\hline Source & Nom du navire & Maître & Propriétaire & Armateur & Destination & Observations diverses \\
\hline $\begin{array}{l}\text { Teuleron } \\
1641,9 / 4\end{array}$ & $\begin{array}{l}\text { Le Gaston } 100 \\
\text { tx. }\end{array}$ & G. Joubert & - & $\begin{array}{l}\text { A. Chaffau pour } \\
\text { la Cie de la N.F. }\end{array}$ & Tadoussac. & - - \\
\hline $\begin{array}{l}\text { Teuleron } \\
1641,9 / 4\end{array}$ & $\begin{array}{l}\text { La Charlotte, } \\
8 \text { tx. }\end{array}$ & V. Achard. & - - & $\begin{array}{l}\text { Id. pour la Cie } \\
\text { de Miscou. }\end{array}$ & $\begin{array}{l}\text { Miscou et la pê- } \\
\text { che. }\end{array}$ & \\
\hline $\begin{array}{l}\text { Teuleron } \\
1641,7 / 5\end{array}$ & $\begin{array}{l}\text { Le René des } \\
\text { Sables } 120 \mathrm{tx} .\end{array}$ & N. Pernet & - - & $\begin{array}{l}\text { Id. pr la Cie de } \\
\text { la N.F. }\end{array}$ & Québec. & - \\
\hline $\begin{array}{l}\text { Teuleron } \\
1641,27 / 3\end{array}$ & Le St François & - & - - & Cl. de Razilly & Acadie. & - \\
\hline $\begin{array}{l}\text { Teuleron } \\
1641,2 / 4 .\end{array}$ & Le St Pierre & M. Duhalde & $\begin{array}{l}\text { J. Tuffet. } \\
\text { P. Desportes. }\end{array}$ & les propriétaires & $\begin{array}{l}\text { Cap Breton \& } \\
\text { pêche. }\end{array}$ & - - \\
\hline
\end{tabular}

1642

\begin{tabular}{|c|c|c|c|c|c|c|}
\hline $\begin{array}{l}\text { Teuleron } \\
1642,16 / 5\end{array}$ & Le St Elie 150 tx & P. Sorut. & E. Leborgne. & Ch. de Menou & $\begin{array}{l}\text { Acadie, La } \\
\text { Heve. }\end{array}$ & - \\
\hline $\begin{array}{l}\text { Teuleron } \\
1642,21 / 9\end{array}$ & La Vierge $120 \mathrm{tx}$ & H. Bourget & E. Leborgne & Ch. de Menou & $\begin{array}{l}\text { Acadie, La He- } \\
\text { ve, Port Royal. }\end{array}$ & $\begin{array}{l}\text { Parti de L.R. le } 25 / 9 \text {, } \\
\text { arr. le } 5 / 12 \text { a Port } \\
\text { Royal, prend part au } \\
\text { combat des forts de } \\
\text { La Tour et Port Roy- } \\
\text { al. Ret. L.R. fin } 1643 \\
\text { (B } 5656.9 / 11644)\end{array}$ \\
\hline $\begin{array}{l}\text { Cherbonnier, } \\
1642,5 / 4 \text {. }\end{array}$ & - - & & - & $\begin{array}{l}\text { G. Desjardins } \\
\text { pr Ch. de St Es- } \\
\text { tienne. }\end{array}$ & $\begin{array}{l}\text { Acadie, riv. St } \\
\text { Jean }\end{array}$ & (D $0000, \quad$ Y \\
\hline $\begin{array}{l}\text { Teuleron } \\
1642,22 / 3\end{array}$ & & J. Decombes & $\begin{array}{l}\text { A. Tuffet \& } \\
\text { P. Desports }\end{array}$ & $\begin{array}{l}\text { L. Gaigneur } \\
\text { J. Pépin }\end{array}$ & $\begin{array}{l}\text { Cap Breton et } \\
\text { pêche }\end{array}$ & \\
\hline $\begin{array}{l}\text { Teuleron } \\
1642,18 / 4\end{array}$ & $\begin{array}{l}\text { L'Espérance de } \\
\text { St Gilles } 90 \mathrm{tx} .\end{array}$ & H. Langevin & - & $\begin{array}{l}\text { A. Cheffault pr } \\
\text { la Cie de la N.F. }\end{array}$ & $\begin{array}{l}\text { Miscou \& } \mathrm{Ta}- \\
\text { doussac. }\end{array}$ & $\begin{array}{l}\text { Quittances après ret. } \\
\text { en fin d'année. }\end{array}$ \\
\hline Id. & $\begin{array}{l}\text { Le St Nicolas de } \\
\text { St Gilles, } 80 \text { tx. }\end{array}$ & J. Richard & $\ldots$ & Id. & Québec & - \\
\hline Id. & $\begin{array}{l}\text { Le St Pierre de } \\
\text { Dieppe, } 80 \text { tx. }\end{array}$ & G. Fincard. & $\ldots$ & Id. . & Id. & 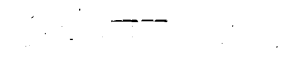 \\
\hline Id. & $\begin{array}{l}\text { Le St François, } \\
\text { de L.R. } 100 \text { tx. }\end{array}$ & J. Barraud & - & Id. & Id. & $\cdots-$ \\
\hline
\end{tabular}




\begin{tabular}{|c|c|c|c|c|c|c|}
\hline $\begin{array}{l}\text { Cherbonnier } \\
1643,8 / 4\end{array}$ & $\begin{array}{l}\text { Le St. Clément } \\
120 \text { tx. }\end{array}$ & J. Guyonncau & & $\begin{array}{l}\text { G. Desjardins } \\
\text { pr Ch. de St Es- } \\
\text { tienne. }\end{array}$ & $\begin{array}{l}\text { Acadie, riv. } \\
\text { St Jean. }\end{array}$ & \\
\hline $\begin{array}{l}\text { Teuleron } \\
1643,8 / 4\end{array}$ & $\begin{array}{l}\text { Le St François } \\
\text { de L.R. } 130 \text { tx. }\end{array}$ & J. Barraud & - & $\begin{array}{l}\text { A. Cheffault pr } \\
\text { la Cie de la N.F. } \\
\text { \& Miscou. }\end{array}$ & $\begin{array}{l}\text { Miscou } \& \mathrm{Ta}- \\
\text { doussac }\end{array}$ & - \\
\hline $\begin{array}{l}\text { Teuleron } \\
1643,27 / 4 \text {. }\end{array}$ & $\begin{array}{l}\text { La Madeleine } \\
\text { d'Olonne, } 80 \mathrm{tx} .\end{array}$ & J. Jouet & -- & Id. & $\begin{array}{l}\text { Tadoussac } \& \\
\text { Québec }\end{array}$ & - - \\
\hline $\begin{array}{l}\text { Teuleron } \\
1643,27 / 4\end{array}$ & $\begin{array}{l}\text { La Marie } \\
\text { Dieppe } 86 \\
\text { tx. }\end{array}$ & P. Metifeu & - & $\begin{array}{l}\text { A. Cheffault pr } \\
\text { la Cie de la N.F. } \\
\text { et Miscou. }\end{array}$ & $\begin{array}{l}\text { Tadoussac et } \\
\text { Québec. }\end{array}$ & - \\
\hline $\begin{array}{l}\text { Teuleron } \\
1643,21 / 3\end{array}$ & Le Petit St Jean & J. Boucher & $\begin{array}{l}\text { A. Tuffet } \\
\text { L. Gaigneur } \\
\text { J. Pépin } \\
\end{array}$ & les propriétaires & $\begin{array}{l}\text { Cap Breton et } \\
\text { pêche. }\end{array}$ & - \\
\hline \multicolumn{7}{|c|}{1644} \\
\hline $\begin{array}{l}\text { Teuleron } \\
1644,19 / 4\end{array}$ & $\begin{array}{l}\text { Le Dauphin, } \\
\text { L.R. } 200 \text { tx. }\end{array}$ & J. Gandouin & - & A. Cheffault & $\begin{array}{l}\text { Miscou \& Ta- } \\
\text { doussac. }\end{array}$ & $\begin{array}{l}\text { Quit. après ret. en } \\
\text { fin d'année. }\end{array}$ \\
\hline Id. & $\begin{array}{l}\text { Le St Clément } \\
120 \text { tx. }\end{array}$ & J. Guyonneau. & G. Desjardins & Id & $\begin{array}{l}\text { Tadoussac \& } \\
\text { Québec }\end{array}$ & $\begin{array}{l}\text { Id. Avaries dans le } \\
\text { St Laurent (B 5656) }\end{array}$ \\
\hline Id. & $\begin{array}{l}\text { La Vierge de } \\
\text { L.R. } 120 \text { tx. }\end{array}$ & H. Bourget & E. Leborgne & Id. & Id. & - - \\
\hline $\begin{array}{l}\text { Teuleron } \\
1644,16 / 7\end{array}$ & $\begin{array}{l}\text { Le Grand Car- } \\
\text { dinal } 200 \text { tx. }\end{array}$ & P. Sauvic & Id. & Ch. de Menou & $\begin{array}{l}\text { Acadie, Port } \\
\text { Royal }\end{array}$ & $\begin{array}{l}\text { Reste en Acadie jus- } \\
\text { qu'en } 1645 \text {, combat } \\
\text { au fort La Tour (B } \\
56568 / 11 / 16 \text { ). }\end{array}$ \\
\hline $\begin{array}{l}\text { В } 5656 \\
1645,3 / 1\end{array}$ & La Jeanne & P. Metifeu & - & Id. & $\begin{array}{l}\text { Retour d'Aca- } \\
\text { die. }\end{array}$ & $\begin{array}{l}\text { Nav. confisqué dép. } \\
\text { de Port Royal le } \\
\text { 20/11, arr. LR le } \\
\text { 29/12. }\end{array}$ \\
\hline $\begin{array}{l}\text { Teuleron } \\
1644,30 / 3\end{array}$ & $\begin{array}{l}\text { Le Petit St Pier- } \\
\text { re, } 70 \text { tx. }\end{array}$ & P. Boileau & $\begin{array}{l}\text { A. Tuffet } \\
\text { A. Duchanin } \\
\text { D. Detchverry. }\end{array}$ & les propriétaires & $\begin{array}{l}\text { Cap Breton et } \\
\text { pêche. }\end{array}$ & - \\
\hline
\end{tabular}


1645

\begin{tabular}{|c|c|c|c|c|c|c|}
\hline Source & Nom du navire & Maître & Propriétaire & Armateur & Destination & Observations diverses \\
\hline $\begin{array}{l}\text { Teuleron } \\
1645,31 / 12 \text {. }\end{array}$ & La Vierge & $\begin{array}{l}\text { P. de Bouguy- } \\
\text { onne }\end{array}$ & E. Leborgne & - & - & $\begin{array}{l}\text { Voyage connu seule- } \\
\text { ment par une at- } \\
\text { testation. }\end{array}$ \\
\hline $\begin{array}{l}\text { Teuleron } \\
1645,27 / 3\end{array}$ & $\begin{array}{l}\text { L'Amitié de LR } \\
200 \mathrm{tx} .\end{array}$ & $\begin{array}{l}\text { C. Pénigault dit } \\
\text { Matha }\end{array}$ & - & E. Leborgne & $\begin{array}{l}\text { Acadie Port } \\
\text { Royal ou La } \\
\text { Heve. }\end{array}$ & - \\
\hline $\begin{array}{l}\text { Teuleron } \\
1645,16 / 8\end{array}$ & $\begin{array}{l}\text { L'Espérance de } \\
\text { Źlande } 230 \mathrm{tx} .\end{array}$ & - & - & E. Lebơrgne & $\begin{array}{l}\text { Acadie Port } \\
\text { Royal }\end{array}$ & $\begin{array}{l}\text { Quittances après re- } \\
\text { tour en } 1646 \text {. }\end{array}$ \\
\hline $\begin{array}{l}\text { Moreau } \\
1645,12 / 4\end{array}$ & L'Etoile $50 \mathrm{tx}$. & J. Thibaud & $\begin{array}{l}\text { G. Guignard sr } \\
\text { du fort ste Anne } \\
\text { et autres }\end{array}$ & Le propriétaire & Cap Breton & $\begin{array}{l}\text { Le navire va à Cap } \\
\text { Breton (Teuleron } \\
1645,5 / 5) \text {. }\end{array}$ \\
\hline $\begin{array}{l}\text { Teuleron } \\
1645,5 / 5\end{array}$ & $\begin{array}{l}\text { Le St Joseph, } \\
300 \text { tx. }\end{array}$ & $\begin{array}{l}\text { J. Pacquier mai- } \\
\text { tre, Prevost Cne }\end{array}$ & N. Denys & N. Denys & Miscou et pêche & - \\
\hline \multicolumn{7}{|c|}{1646} \\
\hline $\begin{array}{l}\text { Teuleron } \\
1646,28 / 6\end{array}$ & $\begin{array}{l}\text { Le Dauphin } \\
\text { d'Amsterdam } \\
200 \text { tx. }\end{array}$ & - & - & E. Leborgne & $\begin{array}{l}\text { Acadie, Port } \\
\text { Royal }\end{array}$ & $\begin{array}{l}\text { Quittance aprés re- } \\
\text { tour le } 29 / 11 \text {. }\end{array}$ \\
\hline $\begin{array}{l}\text { Teuleron } \\
1646,9 / 3\end{array}$ & $\begin{array}{l}\text { Le St Joseph } \\
300 \text { tx. }\end{array}$ & J. Decombes & N. Denys & N. Denys & Miscou et pêche & - \\
\hline $\begin{array}{l}\text { B } 5657 \\
1646,18 / 7 . \\
\text { Id. } \\
\text { Id. }\end{array}$ & $\begin{array}{l}\text { Le Cardinal } \\
\text { La Notre Dame } \\
\text { Le Navire neuf }\end{array}$ & $\begin{array}{l}\text { Le sr de Repen- } \\
\text { tigny } \\
\text { Filly } \\
\text { Godefroy }\end{array}$ & - & $\begin{array}{l}\text { Les habitants de } \\
\text { la (N.F.) }\end{array}$ & $\begin{array}{l}\text { Québec } \\
\simeq-\end{array}$ & - \\
\hline $\begin{array}{l}\text { Moreau } \\
1646,22 / 8\end{array}$ & $\begin{array}{ll}\text { Le Petit } & \text { St } \\
\text { Christophe } & \text { de } \\
\text { LR. } 50 \text { tx. } & \\
\end{array}$ & I. Richard & A. Melot & $\begin{array}{l}\text { O. Le Tardif pr } \\
\text { la gde Cie de la } \\
\text { (N.F.) }\end{array}$ & $\begin{array}{l}\text { Québec ou Ta- } \\
\text { Tadoussac. }\end{array}$ & - \\
\hline
\end{tabular}




\begin{tabular}{|c|c|c|c|c|c|c|}
\hline $\begin{array}{l}\text { Teuleron } \\
1647,27 / 4\end{array}$ & $\begin{array}{l}\text { Le St Jean hol- } \\
\text { landais } 200 \mathrm{tx} \text {. }\end{array}$ & - & & E. Leborgne & $\begin{array}{l}\text { Acadie riv. St } \\
\text { Jean }\end{array}$ & - \\
\hline $\begin{array}{l}\text { Teuleron } \\
1647,3 / 6\end{array}$ & $\begin{array}{l}\text { Le Bon ou le } \\
\text { S. François de } \\
\text { Dieppe, } 90 \text { tx. }\end{array}$ & - & - & $\begin{array}{l}\text { P. LeGardeur } \\
\text { de Repentigny }\end{array}$ & Québec & -- \\
\hline $\begin{array}{l}\text { Teuleron } \\
1647,6 / 6\end{array}$ & $\begin{array}{l}\text { La Marguerite } \\
\text { de LR., } 70 \text { tx. }\end{array}$ & - & - & $\begin{array}{l}\text { P. LeGardeur } \\
\text { N. Juchereau }\end{array}$ & Id. & - \\
\hline \multicolumn{7}{|c|}{1648} \\
\hline $\begin{array}{l}\text { Teuleron } \\
1648,5 / 6 .\end{array}$ & $\begin{array}{l}\text { Le S. Georges, } \\
70 \text { tx. }\end{array}$ & - & Cte du Dognon & $\begin{array}{l}\text { N. Juchereau } \\
\text { commis Gal. des } \\
\text { habts de la N.F. }\end{array}$ & Tadoussac & - \\
\hline $\begin{array}{l}\text { Michelon } \\
1648,1 / 7 \text {. }\end{array}$ & $\begin{array}{l}\text { Le Grand Car- } \\
\text { dinal }\end{array}$ & - & - & & Québec & - \\
\hline Id. & La Notre-Dame & - & - & Id. & Id. & - \\
\hline Id. & Le S. Sauveur & - & - & & Id. & - \\
\hline $\begin{array}{l}\text { Teuleron } \\
1648,5 / 3\end{array}$ & $\begin{array}{l}\text { La Notre-Dame } \\
\text { de Miscou }\end{array}$ & J. Paquier & N. Denys & Le propriétaire & Miscou et pêche & - \\
\hline $\begin{array}{l}\text { Teuleron } \\
1648,1 / 5 .\end{array}$ & $\begin{array}{l}\mathrm{La} \text { Verve de } \\
\mathrm{Middleb} \text { o u rg } \\
260 \text { tx. }\end{array}$ & - & - & E. Leborgne & Acadie & - - \\
\hline \multicolumn{7}{|c|}{1649} \\
\hline $\begin{array}{l}\text { Michelon } \\
1649,21 / 3 .\end{array}$ & $\begin{array}{l}\text { Le S. Sauveur } \\
\text { de Québec } 150 \\
\text { tx. }\end{array}$ & J. Decombes & $\begin{array}{l}\text { J. Juchereau } \\
\text { J. Chaujon } \\
\text { P. Teuleron }\end{array}$ & Les propriétaires & Québec et pêche & - \\
\hline $\begin{array}{l}\text { Teuleron } \\
1649,28 / 5\end{array}$ & $\begin{array}{l}\text { Le Bon François } \\
\text { de Dieppe, } 90 \text { t. }\end{array}$ & - & - & J. Godefroy & Québec & - \\
\hline $\begin{array}{l}\text { Teuleron } \\
1649,29 / 5\end{array}$ & $\begin{array}{l}\text { Le Gd Cardinal, } \\
300 \text { tx. La No- } \\
\text { tre-Dame, } 200 \text { t. }\end{array}$ & - & - - & $\begin{array}{l}\text { J. Godefroy } \\
\text { et J. Juchereau } \\
\text { pr la commu- } \\
\text { nauté de Québec }\end{array}$ & $\begin{array}{l}\text { Québec } \\
\text { Id. }\end{array}$ & - \\
\hline
\end{tabular}


1650

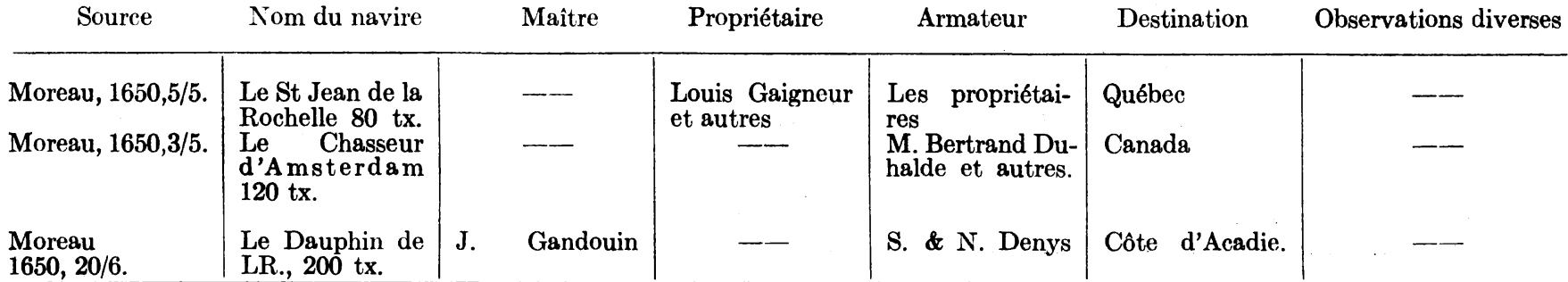

1651

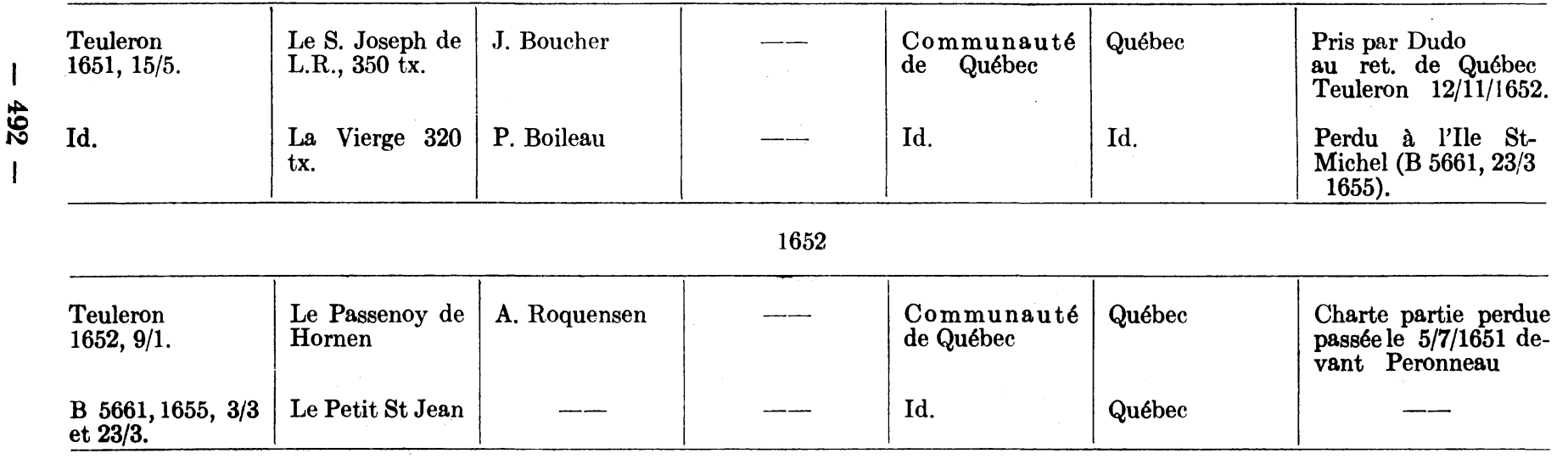

1653

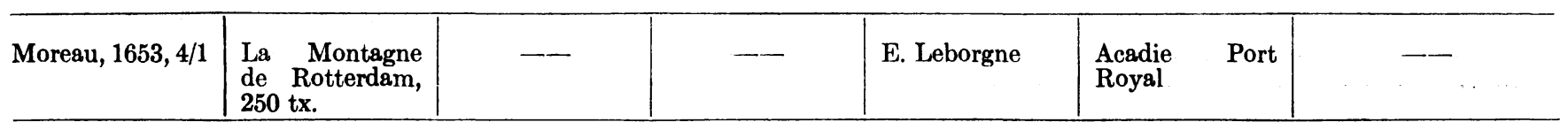




\begin{tabular}{|c|c|c|c|c|c|c|}
\hline $\begin{array}{l}\text { Cherbonnier, } \\
1654,8 / 3 \text {. }\end{array}$ & $\begin{array}{l}\text { Le Chateau fort } \\
200 \text { tx. }\end{array}$ & - - & $\begin{array}{l}\text { E. Leborgne } \\
\text { A. Lucas } \\
\text { P. Pollart } \\
\end{array}$ & - & $\begin{array}{l}\text { Acadie } \\
\text { Royal }\end{array}$ & - \\
\hline \multicolumn{7}{|c|}{1655} \\
\hline $\begin{array}{l}\text { B } 5661 \\
1655,8 / 11 . \\
\text { Id. }\end{array}$ & $\begin{array}{l}\text { Le Petit Fran- } \\
\text { cois, } 50 \text { tx. } \\
\text { Le Chat Bouque }\end{array}$ & P. Delafond & Fr. Peron & Le propriétaire & $\begin{array}{r}\text { Québec } \\
\qquad- \\
\end{array}$ & $\begin{array}{l}\text { Ne réussit pas a at } \\
\text { teindre Québec. }\end{array}$ \\
\hline \multicolumn{7}{|c|}{1656} \\
\hline $\begin{array}{l}\text { Moreau } \\
1656,10 / 4 .\end{array}$ & Le René, 80 tx. & - & - & A. Grignon & Québec & - - \\
\hline B $5662,1656,66 / 3$. & $?$ & - & - & E. Leborgne & - & - \\
\hline $\begin{array}{l}\text { Cherbonnier, } \\
1656,12 / 4 \text {. }\end{array}$ & $\begin{array}{l}\text { Le Taureau } 150 \\
\text { tx. }\end{array}$ & E. Tadourneau & Fr. Peron & Le propriétaire & Id. & - - \\
\hline $\begin{array}{l}\text { Moreau, } \\
1656,8 / 4 .\end{array}$ & $\begin{array}{l}\text { La Fortune } 100 \\
\text { tx. }\end{array}$ & E. Raymond & Auboyneau & $\begin{array}{l}\text { J. Pépin } \\
\text { P. Thévenin }\end{array}$ & Id. & - \\
\hline \multicolumn{7}{|c|}{1657} \\
\hline $\begin{array}{l}\text { Moreau } \\
1657,20 / 1\end{array}$ & $\begin{array}{l}\mathrm{L} \text { es A r m es } \\
\text { d'A msterdam } \\
250 \mathrm{tx} \text {. }\end{array}$ & - - & - & $\begin{array}{l}\text { P. Gaigneur } \\
\text { J. Massé }\end{array}$ & Id. & - \\
\hline $\begin{array}{l}\text { Cherbonnier, } \\
1657,4 / 4 \text {. }\end{array}$ & Le Taureau & E. Tadourneau & Fr. Peron & $\begin{array}{l}\text { Les proprie- } \\
\text { taires }\end{array}$ & Québec & - \\
\hline $\begin{array}{l}\text { Cherbonnier } \\
1657,24 / 2 \text {. }\end{array}$ & La Vierge & F. Marot & - & - & Id. & - \\
\hline $\begin{array}{l}\text { Moreau } \\
1657,21 / 10 .\end{array}$ & $?$ & P. Jamain & A. Petit & - & Acadie & - \\
\hline
\end{tabular}


1658

\begin{tabular}{|c|c|c|c|c|c|c|}
\hline Source & Nom du navire & Maître & Propriétaire & Armateur & Destination & Observations diverses \\
\hline $\begin{array}{l}\text { Cherbonnier } \\
1658,17 / 5 \text { et B } \\
5663 \text { no } 226 \text {. }\end{array}$ & Le Taureau & E. Tadourneau & Fr. Peron & Le propriétaire & Québec & - \\
\hline $\begin{array}{l}\text { Teuleron } \\
1658,19 / 2 .\end{array}$ & Le St Joseph & Marot & - & - - & - - & - - \\
\hline $\begin{array}{l}\text { Teuleron } \\
1658,6 / 5 \text { et } 17 / 4 \text {. }\end{array}$ & $\begin{array}{l}\text { Le Prince Guil- } \\
\text { laume } 150 \mathrm{tx} \text {. }\end{array}$ & - - & - & - & $\begin{array}{l}\text { Nouvelle Fran- } \\
\text { ce }\end{array}$ & - \\
\hline
\end{tabular}

1659

\begin{tabular}{|c|c|c|c|c|c|c|}
\hline $\begin{array}{l}\text { Moreau } \\
1659,26 / 5 .\end{array}$ & Id. & - & - & $\begin{array}{l}\text { J. Pépin \& au- } \\
\text { tres }\end{array}$ & Acadie & - \\
\hline $\begin{array}{l}\text { Moreau } \\
1659,28 / 6 .\end{array}$ & $\begin{array}{l}\text { Le St André } 300 \\
\text { tx. }\end{array}$ & G. Poullet & J. Nezereau & J. Mousnier & Quebec & - \\
\hline $\begin{array}{l}\text { Cherbonnier } \\
1659,14 / 3 \text {. }\end{array}$ & $\begin{array}{l}\text { Le Sacrifice } \\
\text { d'Abraham, } 300 \\
\text { tx. }\end{array}$ & - & - & - & Id. & \\
\hline
\end{tabular}

1660

\begin{tabular}{l|l|l|l|l|l|l}
\hline $\begin{array}{l}\text { Juppin } \\
\text { 1660, 27/2. }\end{array}$ & $\begin{array}{l}\text { Le St Jean } \\
100 \text { tx. }\end{array}$ & E. Raymond & $\begin{array}{l}\text { J. Gitton \& au- } \\
\text { tres }\end{array}$ & $\begin{array}{l}\text { Les propriétai- } \\
\text { res }\end{array}$ & $\begin{array}{l}\text { N.F. \& N. An- } \\
\text { gleterre }\end{array}$ & \\
& & & \\
\hline
\end{tabular}


1661

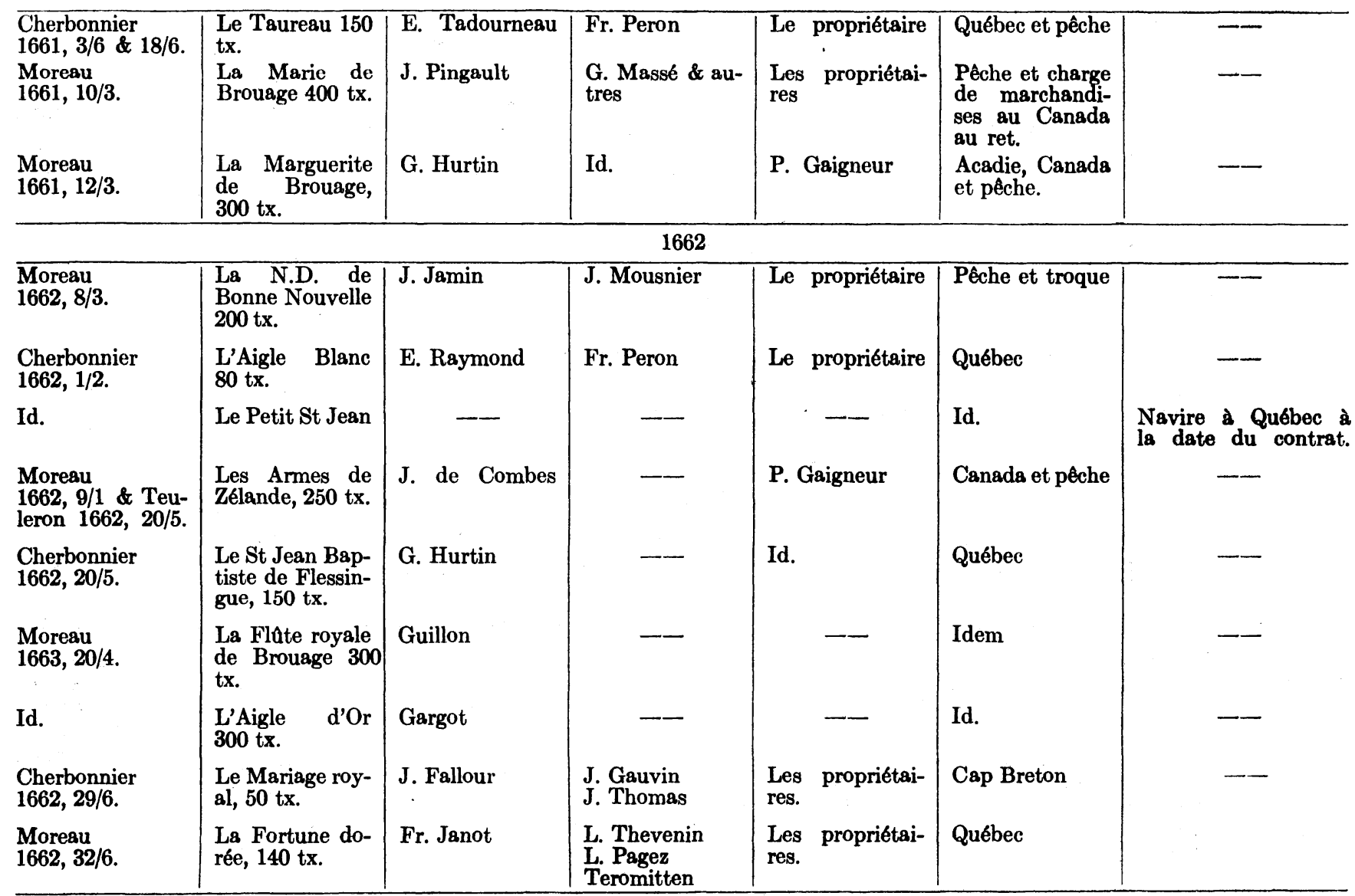


1663

\begin{tabular}{|c|c|c|c|c|c|c|}
\hline Source & Nom du navire & Maître & Propriétaire & Armateur & Destination & Observations diverses \\
\hline $\begin{array}{l}\text { Moreau } \\
1662,8 / 12, \\
\text { Teul. } 1663,23 / 4 \text {. }\end{array}$ & $\begin{array}{l}\text { Le Phénix de } \\
\text { Flessingue } 200 \\
\text { tx. }\end{array}$ & - & - & P. Gaigneur & $\begin{array}{l}\text { Canada, Québec } \\
\text { et pêche }\end{array}$ & - \\
\hline $\begin{array}{l}\text { Cherbonnier } \\
1664,26 / 3 \text { et } \\
\text { B 5664 no } 138\end{array}$ & Le Taureau & E. Raymon & Fr. Peron & Le propriétaire & Québec & $\begin{array}{l}\text { Dép. } 10 \text { Mai, arr. } \\
\text { Québec } 24 \text { Juillet Ret. } \\
19 \text { sept. - } 20 \text { Octobre. }\end{array}$ \\
\hline
\end{tabular}

1664

\begin{tabular}{|c|c|c|c|c|c|c|}
\hline $\begin{array}{l}\text { Teuleron } \\
1644,11 / 2 .\end{array}$ & $\begin{array}{l}\text { L'Aigle blanc } \\
\text { de Flessingue }\end{array}$ & A. Chaviteau & - & P. Gaigneur & Canada & - \\
\hline $\begin{array}{l}\text { Cherbonnier, } \\
\text { 1664, 22/4. }\end{array}$ & $180 \mathrm{tx}$. & & & & & \\
\hline $\begin{array}{l}\text { Cherbonnier, } \\
1644,14 / 2 \text {, et } 5 / 4 \text {. }\end{array}$ & $\begin{array}{l}\text { Le Noir d'Am- } \\
\text { sterdam } 100 \mathrm{tx} \text {. }\end{array}$ & P. Fillye & - & $\begin{array}{l}\text { la compagnie de } \\
\text { Rouen }\end{array}$ & $\begin{array}{l}\text { Québec et peche } \\
\text { retour a Dieppe }\end{array}$ & $\begin{array}{l}\text { Liste de passagers } \\
\text { B } 5665 \text { no } 110 \text {. }\end{array}$ \\
\hline
\end{tabular}

1665

\begin{tabular}{|c|c|c|c|c|c|c|}
\hline $\begin{array}{l}\text { Teuleron } \\
1665,28 / 2\end{array}$ & $\begin{array}{l}\text { Le Vieux Sime- } \\
\text { on de Durker- } \\
\text { dam 200.tx. }\end{array}$ & - & - & P. Gaigneur & Québec & - \\
\hline $\begin{array}{l}\text { Teuleron } \\
1665,22 / 3\end{array}$ & $\begin{array}{l}\text { Le Cat de Hol- } \\
\text { lande }\end{array}$ & - - & - & Id. & Id. & - \\
\hline $\begin{array}{l}\text { B } 5666 \text { no } 85 \text {, } \\
1665,22 / 11\end{array}$ & $\begin{array}{l}\text { Le Jardin de } \\
\text { Hollande }\end{array}$ & Ce Desboniges & - - & Le Roi & Id. & - \\
\hline $\begin{array}{l}\text { B } 5666 \text { no. 107, } \\
1665,23 / 11 \text {. }\end{array}$ & La Paix & J. Guillon & - & Id. & Id. & $\begin{array}{l}\text { Naufragé au ret. de } \\
\text { Québec. }\end{array}$ \\
\hline
\end{tabular}




\begin{tabular}{|c|c|c|c|c|c|c|c|}
\hline $\begin{array}{l}\text { Teuleron } \\
1666,16 / 1\end{array}$ & $\begin{array}{l}\text { Le St Joseph } \\
300 \text { tx. }\end{array}$ & J. Tadourneau & $\begin{array}{l}\text { J. Mousnier } \\
\text { P Grigneur }\end{array}$ & P. Gaigneur & Québec & & - \\
\hline $\begin{array}{l}\text { Teuleron } \\
1666,26 / 2 .\end{array}$ & La Paix 180 tx. & M. Fory & J. Depont & $\begin{array}{l}\text { P. Gaigneur et } \\
\text { J. Le Royer }\end{array}$ & Québec et pêche & & - \\
\hline $\begin{array}{l}\text { Teuleron } \\
1666,6 / 5\end{array}$ & $\begin{array}{l}\text { Le Paon de Hol- } \\
\text { lande } 200 \text { tx. }\end{array}$ & A. Chaviteau & - - & P. Gaigneur & Québec & & - \\
\hline $\begin{array}{l}\text { Teuleron } \\
1666,27 / 5\end{array}$ & $\begin{array}{l}\text { La Fortune } \\
\text { Blanche d'Am- } \\
\text { sterdam } 200 \text { tx. }\end{array}$ & V. de Neuville & - & Id. & Id. & & - \\
\hline $\begin{array}{l}\text { B } 5667 \text { no. } \\
1666,17 / 5 .\end{array}$ & $\begin{array}{l}\text { Le St Jean Bap- } \\
\text { tiste de Dieppe }\end{array}$ & Fillie & Cie des Indes & Id. & Id. & & - \\
\hline $\begin{array}{l}\text { Teuleron } \\
1666,14 / 5\end{array}$ & L'Amitié 50 tx. & N. Graton & - & J. Lamothe & $\begin{array}{l}\text { Québec, ret. a } \\
\text { Amsterdam ou } \\
\text { La Rochelle }\end{array}$ & & - \\
\hline Teuleron 1666 & $\begin{array}{l}\text { La Neptune } 100 \\
\text { tx. }\end{array}$ & -- & $\begin{array}{l}\text { E. Leborgne et } \\
\text { autres }\end{array}$ & $\begin{array}{l}\text { Les propriétai- } \\
\text { res }\end{array}$ & $\begin{array}{l}\text { Acadie } \begin{array}{c}\text { Mace- } \\
\text { doine et peche. }\end{array} \\
\end{array}$ & & -- \\
\hline \multicolumn{8}{|c|}{1667} \\
\hline B 5668 no. 223. & Le Clerbault & - & C. Bergier & - & - & Liste & de passagers. \\
\hline $\begin{array}{l}\text { Teuleron, } 1667 \text {, } \\
13 / 4 \text { et } 17 / 5 \text {. }\end{array}$ & Le St Philippe & P. Gentet & Pagez et autres & P. Gaigneur & Québec & & - \\
\hline $\begin{array}{l}\text { Teuleron } \\
1667,30 / 4\end{array}$ & $\begin{array}{l}\text { L'Orange de Ni- } \\
\text { eudam }\end{array}$ & - & - - & Id. & Id. & & - \\
\hline $\begin{array}{l}\text { Teuleron } \\
1667,20 / 5\end{array}$ & $\begin{array}{l}\text { Le Prophète } \\
\text { Elie } 260 \text { tx. }\end{array}$ & - & E. de Baussay & Id. & Id. & & - \\
\hline $\begin{array}{l}\text { Teuleron } \\
1667,1 / 7\end{array}$ & La Catherine & J. Chaillé & $\begin{array}{l}\text { J. Depont \& } \\
\text { P. Faneuil }\end{array}$ & Id. & Id. & & - \\
\hline $\begin{array}{l}\text { Teuleron } \\
1667,16 / 5\end{array}$ & $\begin{array}{l}\text { Le Petit Agneau } \\
\text { Agneau } 160 \text { tx. }\end{array}$ & - & - & - & - & & - \\
\hline $\begin{array}{l}\text { Teuleron } \\
1667,5 / 5\end{array}$ & $\begin{array}{l}\text { La Nouvelle } \\
\text { France } 250 \text { tx. }\end{array}$ & A. Chaviteau & P. Grigneur & Id. & $\begin{array}{l}\text { Québec et la pe- } \\
\text { che. }\end{array}$ & & \\
\hline $\begin{array}{l}\text { Teuleron } \\
1667,14 / 4\end{array}$ & $\begin{array}{l}\text { L8 Constance } \\
300 \mathrm{tx} \text {. }\end{array}$ & A. Pasquier & Id. & Id. & Id. & & - \\
\hline
\end{tabular}


1668

\begin{tabular}{|c|c|c|c|c|c|c|}
\hline Source & Nom du navire & Maître & Propriétaire & Armateur & Destination & Observations diverses \\
\hline $\begin{array}{l}\text { Teuleron } \\
1668,15 / 6 .\end{array}$ & Le Don de Dieu & S. Martel & $\begin{array}{l}\text { S. Martel et au- } \\
\text { tres }\end{array}$ & Le propriétaire & Canada & - \\
\hline $\begin{array}{l}\text { Teuleron } \\
1668,6 \text { et } 18 / 6 \text {. }\end{array}$ & $\begin{array}{l}\text { La Ste Anne de } \\
\text { Holstein }\end{array}$ & - & - & P. Gaigneur & Québec & - - \\
\hline Id. & $\begin{array}{l}\text { La Nouvelle } \\
\text { France }\end{array}$ & - & P. Gaigneur & Id. & Id. & - \\
\hline B 5669 No 7 & $\begin{array}{l}\text { Le St Antoine } \\
\text { l'Espagnol }\end{array}$ & A. Gosse & - & Le Roi & - & - \\
\hline
\end{tabular}

1

$\infty$

\begin{tabular}{|c|c|c|c|c|c|c|}
\hline $\begin{array}{l}\text { B } 5669 \text { no } 41670 \text {, } \\
9 / 1 \text { \& Teuleron } \\
1669,13 / 4 \text {. }\end{array}$ & Le Chat 250 tx. & T. Chaillaud & 一 & Ch. Aubert & Québec & $\begin{array}{l}\text { Dep. } 11 \text { Juin, arr. } \\
\text { Rivière de Québec } 20 \\
\text { Aout. Ret. } 28 \text { sept. } \\
12 \text { décembre. }\end{array}$ \\
\hline $\begin{array}{l}\text { B } 5669 \text { no } 5 \\
1670,10 / 1 .\end{array}$ & L'Espérance & J. Masson & - & - - & Id. & $\begin{array}{l}\text { Dep. 30 Juin, arr. } \\
\text { Québec } 15 \text { Sept. Ret. } \\
10 \text { Nov. - } 15 \text { Déc. }\end{array}$ \\
\hline $\begin{array}{l}\text { Teuleron } \\
1669,8 / 5\end{array}$ & $\begin{array}{l}\text { Le St Pierre de } \\
\text { Hambourg } \\
300 \text { tx. }\end{array}$ & - & - - & P. Gaigneur & Id. & - \\
\hline $\begin{array}{l}\text { Teuleron } \\
1669,6 / 4\end{array}$ & $\begin{array}{l}\text { Le Pot de Beur- } \\
\text { re } 300 \text { tx. } \\
\text { de Horn }\end{array}$ & - & - & Id. & Id. & - \\
\hline
\end{tabular}




\begin{tabular}{|c|c|c|c|c|c|c|}
\hline $\begin{array}{l}\text { В } 5669 \text { nos } 38 \text { \& } \\
1981670,26 / 4 . \&\end{array}$ & $\begin{array}{l}\text { L'Hélène de } \\
\text { Flessingue }\end{array}$ & A. Chaviteau & - & A. Petit & Qứebec & $\begin{array}{l}\text { Dép. le } 22 \text { Mai arr. } \\
\text { Québec le } 18 \text { Aout. }\end{array}$ \\
\hline $\begin{array}{l}\text { Teuleron } \\
1670,20 / 3\end{array}$ & & & - & & & $\begin{array}{l}\text { Dép. de Québec le } 8 \\
\text { Octobre. }\end{array}$ \\
\hline $\begin{array}{l}\text { B } 5669 \text { nos } 46 \text { et } \\
2141670,20 / 3 \text {. }\end{array}$ & Le St Pierre & J. Boutin & - & P. Gaigneur & Id. & $\begin{array}{l}\text { Dép. le } 19 \text { Juin arr. } \\
\text { Québec le } 21 \text { Sept. } \\
\text { Ret. La Rochelle le } \\
5 \text { Décembre. }\end{array}$ \\
\hline $\begin{array}{l}\text { B } 5669 \text { no } 129, \\
1670,25 / 10 .\end{array}$ & $\begin{array}{l}\text { La Nouvelle } \\
\text { France }\end{array}$ & A. Durand & - & Id. & Id. & $\begin{array}{l}\text { Liste de cargaison de } \\
\text { retour. }\end{array}$ \\
\hline \multicolumn{7}{|c|}{1671} \\
\hline $\begin{array}{l}\text { B } 5670 \text { no } 168 \\
1671,15 / 6 \text { et Teu- } \\
\text { leron } 1671,12 / 6 .\end{array}$ & $\begin{array}{l}\text { L'Espérance } \\
160 \text { tx. }\end{array}$ & F. Thibaud & $\begin{array}{l}\text { J. Gitton et au- } \\
\text { tres. }\end{array}$ & $\begin{array}{l}\text { Les propriétai- } \\
\text { res. }\end{array}$ & Québec & - - \\
\hline $\begin{array}{l}\text { B } 5670 \text { no } 177 \text {, } \\
1671,24 / 6 \text { \& Teu- } \\
\text { leron } 1671,15 / 6 \text {. }\end{array}$ & $\begin{array}{l}\text { Le Prince Mau- } \\
\text { rice }\end{array}$ & P. Heraud & Id. & Id. & Id. & - \\
\hline $\begin{array}{l}\text { B } 6570 \text { no } 180 \\
1671,26 / 6 .\end{array}$ & L'Amitié & P. Gentet & - & - & Id. & - \\
\hline $\begin{array}{l}\text { B } 5670 \text { no } 182, \\
1671,3 / 7 \text {. }\end{array}$ & La Plume d'or & J. Goislin & $\begin{array}{l}\text { Pachot, Perez, } \\
\text { Grignon }\end{array}$ & A. Petit & Québec & $\begin{array}{l}\text { En remplacement de } \\
\text { la Sagesse; armée par } \\
\text { le même \& naufragée } \\
\text { a Nantes. }\end{array}$ \\
\hline $\begin{array}{l}\text { B } 5670 \text { no } 303, \\
1671, \quad 14 / 11 \text { et }\end{array}$ & $\begin{array}{l}\text { La Providence } \\
\text { de St-Jean de- }\end{array}$ & P. d'Artiague & - & - & $\begin{array}{l}\text { Acadie et An- } \\
\text { tilles }\end{array}$ & \\
\hline $\begin{array}{l}\text { Teuleron même } \\
\text { date. }\end{array}$ & Luz & & & . & & \\
\hline $\begin{array}{l}\text { Teuleron } \\
1671,8 / 4 .\end{array}$ & $\begin{array}{l}\text { L'Espérance de } \\
\text { Québec, } 300 \text { tx. }\end{array}$ & - & $\begin{array}{l}\text { Ch. Aubert \& } \\
\text { Ch. Bazire }\end{array}$ & $\begin{array}{l}\text { Les propriétai- } \\
\text { res. }\end{array}$ & Ile percée & - \\
\hline
\end{tabular}


1672

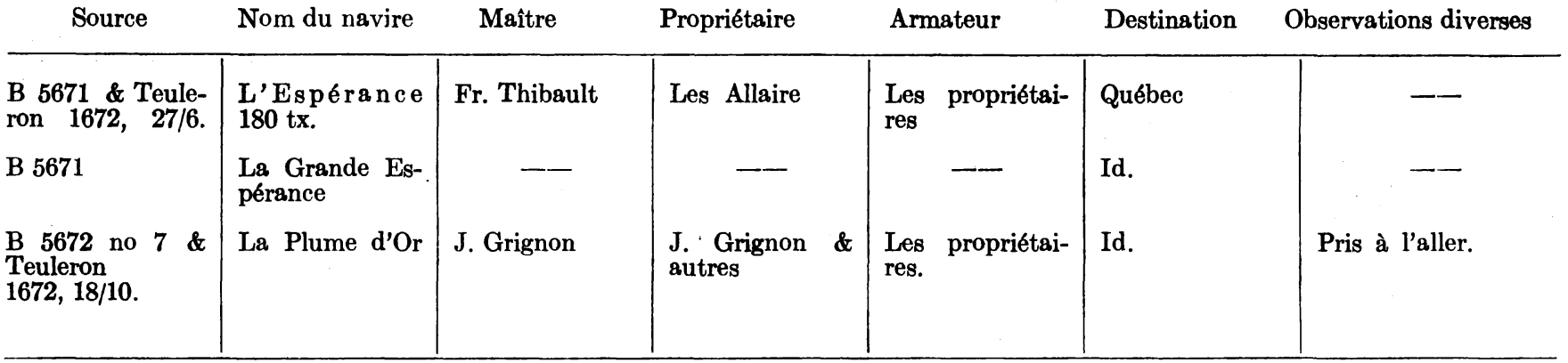

1673

\begin{tabular}{|c|c|c|c|c|c|c|}
\hline $\begin{array}{l}\text { B } 5672 \text { no } 238 \text {, } \\
1673,6 / 5 \text { \& Teu- } \\
\text { leron } 1673,6 / 5 \text {. }\end{array}$ & $\begin{array}{l}\text { Le Prince Mau- } \\
\text { rice } 100 \mathrm{tx} \text {. }\end{array}$ & J. Duhalde & $\begin{array}{l}\text { Les Garos \& } \mathbf{S} . \\
\text { Baston }\end{array}$ & $\begin{array}{l}\text { Les propriétai- } \\
\text { res }\end{array}$ & Québec & - - \\
\hline $\begin{array}{l}\text { B } 5672 \text { no } 240, \\
1673,5 / 6 .\end{array}$ & Le Postillon & P. Thomas & - & - & $\begin{array}{l}\text { Québec et An- } \\
\text { tilles }\end{array}$ & $\begin{array}{l}\text { Dép. le } 11 / 9 \text {, arr. } \\
\text { Québec le } 1 / 10 \text {. Dép. } \\
\text { le } 16 / 11 \text { pour les An- } \\
\text { tilles. }\end{array}$ \\
\hline $\begin{array}{l}\text { Id. no } 243 \\
1673,22 / 6 .\end{array}$ & La Nativité & T. Chaillaud & - & F. Dombourg & Québec & - \\
\hline $\begin{array}{l}\text { Id. no } 246 \\
1673,5 / 7\end{array}$ & L'Espérance & G. Basset & - - & - & Id. & - - \\
\hline $\begin{array}{l}\text { Id. no } 247 \\
1673,6 / 7\end{array}$ & Le Bremois & A. Chaviteau & - & - - & Id. & - \\
\hline $\begin{array}{l}\text { Id. no } 249 \\
1673,3 / 2 .\end{array}$ & La Marianne & J. Monbeuil & - & - & Plaisance & - \\
\hline
\end{tabular}




\begin{tabular}{|c|c|c|c|c|c|c|}
\hline $\begin{array}{l}\text { B } 5673 \text { no } 238 \text {, } \\
1674,19 / 6 \text { \& Teu- } \\
\text { leron } 1674,30 / 6 \text {. }\end{array}$ & $\begin{array}{l}\text { L'Espérance } \\
200 \text { tx. }\end{array}$ & J. Chauvet & $\begin{array}{l}\text { J. Gitton et Ch. } \\
\text { Aubert }\end{array}$ & - & Québec & - \\
\hline $\begin{array}{l}\text { B } 5673 \text { no } 240, \\
1674,21 / 6 \& \text { Teu- } \\
\text { leron } 1674,8 / 5\end{array}$ & $\begin{array}{l}\text { Le M o u t o n } \\
\text { Blanc, } 300 \text { tx. }\end{array}$ & A. Chaviteau & La Cie du Nord & Ch. Aubert & Id. & - \\
\hline $\begin{array}{l}\text { Teuleron } \\
1673,6 / 7 \& 8 / 11\end{array}$ & $\begin{array}{l}\text { Le Lion d'Or, } \\
80 \text { tx. }\end{array}$ & - - & $\begin{array}{l}\text { J. Thomas \& au- } \\
\text { tres }\end{array}$ & Ch. Aubert & $\begin{array}{l}\text { Ile percée puis } \\
\text { Antilles }\end{array}$ & Dép. le $11 / 6$. \\
\hline
\end{tabular}

1675

\begin{tabular}{|c|c|c|c|c|c|c|}
\hline $\begin{array}{l}\text { B } 5674 \text { no } 257, \\
1675,13 / 6 .\end{array}$ & $\begin{array}{l}\text { La Fortune } \\
\text { Blanche }\end{array}$ & N. Noël & A. Perez & - & Québec & -- \\
\hline $\begin{array}{l}\text { B } 5674 \text { no } 259 \\
1675,27 / 6\end{array}$ & $\begin{array}{l}\text { La Dame Anne, } \\
250 \mathrm{tx} .\end{array}$ & Raymond & Les Pagez & - & Id. & - - \\
\hline $\begin{array}{l}\text { B } 5674 \text { no } 260 \text {, } \\
1675,27 / 6 .\end{array}$ & $\begin{array}{l}\text { La Grande Es- } \\
\text { pérance }\end{array}$ & Durand & Ch. Aubert & - & Id. & - - \\
\hline B 5674 no 261, s.d. & La Diane & J. Masson & - & - & Id. & - - \\
\hline $\begin{array}{l}\text { Teuleron } \\
1675,3 / 11\end{array}$ & La Providence & - & - & - & - & - - \\
\hline
\end{tabular}

1676

\begin{tabular}{|c|c|c|c|c|c|c|}
\hline $\begin{array}{l}\text { B } 5675 \text { no } 210 \\
1676,30 / 6\end{array}$ & L'Aigle Noir & P. Gentet & - & - & Québec & - \\
\hline $\begin{array}{l}\text { B } 5675 \text { no } 211 \text { et } \\
\text { Bourot } 1676,10 / 6 \text {. }\end{array}$ & $\begin{array}{l}\text { La Françoise du } \\
\text { Havre } 150 \text { tx. }\end{array}$ & Th. Poitevin & - & - & Id. & - \\
\hline $\begin{array}{l}\text { B } 5675 \text { no } 213 \text {, } \\
1676,20 / 6 .\end{array}$ & $\begin{array}{l}\text { Le Mouton } \\
\text { blanc }\end{array}$ & P. Gravouil & Ch. Aubert & - & Id. & - \\
\hline
\end{tabular}


1677

\begin{tabular}{|c|c|c|c|c|c|c|}
\hline Source & Nom du navire & Maître & Propriétaire & Armateur & Destination & Observations diverses \\
\hline $\begin{array}{l}\text { B } 5676 \text { no } 132, \\
1677,20 / 6 \text { \& Teu- } \\
\text { leron } 1677,1 / 5 \text {. }\end{array}$ & $\begin{array}{l}\text { L'Aigle Noir, } \\
150 \mathrm{tx.}\end{array}$ & J. Chauvet & $\begin{array}{l}\text { J. Gitton \& Fr. } \\
\text { Duprat }\end{array}$ & $\begin{array}{l}\text { Les propriétai- } \\
\text { res }\end{array}$ & $\begin{array}{l}\text { Québec et An- } \\
\text { tilles }\end{array}$ & - - \\
\hline $\begin{array}{l}\text { B } 5676 \text { no } 135 \text {, } \\
1677,6 / 4 .\end{array}$ & $\begin{array}{l}\text { Le Prince Mau- } \\
\text { rice, } 100 \text { tx. }\end{array}$ & E. Mouliot & J. Gitton & - & Canada & - \\
\hline $\begin{array}{l}\text { B } 5676 \text { no } 138 \text {, } \\
1677,14 / 6 .\end{array}$ & $\begin{array}{l}\text { La Grande Es- } \\
\text { pérance }\end{array}$ & A. Durand & - & - - & Canada & - - \\
\hline
\end{tabular}

1678

\begin{tabular}{|c|c|c|c|c|c|c|}
\hline $\begin{array}{l}\text { B } 5677 \text { no } 130, \\
1678,27 / 5 .\end{array}$ & L'Honoré & A. Durand & A. Grignon & - & $\begin{array}{l}\text { Canada et An- } \\
\text { tilles }\end{array}$ & - \\
\hline $\begin{array}{l}\text { B } 5677 \text { no } 134 \text {, } \\
1678,2 / 5 \text {. }\end{array}$ & $\begin{array}{l}\text { La Grande Es- } \\
\text { pérance }\end{array}$ & A. Chaviteau & - & - & Québec & - \\
\hline $\begin{array}{l}\text { B } 5677 \text { no } 135 \text {, } \\
1678,2 / 5 \text {. }\end{array}$ & Le St Joseph & N. Dalton & - & - & Id. & - \\
\hline $\begin{array}{l}\text { B } 5677 \text { no } 132, \\
1678,2 / 5 \text {. }\end{array}$ & Le Mouton & $\underset{\text { Michel }}{\mathrm{J}}-\mathrm{B} \cdot \mathrm{d}$ e S t & Ch. Aubert & - & Id. & - \\
\hline $\begin{array}{l}\text { B } 5677 \text { no } 136, \\
1678,4 / 7 \text {. }\end{array}$ & L'Aigle noir & J. Chauvet & J. Gitton & - - & Id. & - \\
\hline $\begin{array}{l}\text { B } 5677 \text { no } 137, \\
1678,7 / 6 .\end{array}$ & $\begin{array}{l}\text { La F ortune } \\
\text { blanche }\end{array}$ & P. Catalogne & A. Perez & - & Plaisance & - \\
\hline $\begin{array}{l}\text { Teuleron } \\
1678,4 / 4\end{array}$ & $\begin{array}{l}\text { Le Prince Mau- } \\
\text { rice }\end{array}$ & J. Blondet & $\begin{array}{l}\text { J. Gitton \& Fr. } \\
\text { Duprat }\end{array}$ & $\begin{array}{l}\text { Les propriétai- } \\
\text { res }\end{array}$ & $\begin{array}{l}\text { Acsdie et Qué- } \\
\text { bec }\end{array}$ & $\longrightarrow$ \\
\hline
\end{tabular}


1679

\begin{tabular}{|c|c|c|c|c|c|c|c|}
\hline $\begin{array}{l}\text { B } 5678 \text { no } 343 \text {, } \\
1679,17 / 7 .\end{array}$ & L'Honoré & A. Durand & J. Grignon & & - & $\begin{array}{l}\text { Canada et An- } \\
\text { tilles }\end{array}$ & - - \\
\hline $\begin{array}{l}\text { B } 5678 \text { no } 345 \& \\
\text { Teuleron } \\
1679,20 / 7 .\end{array}$ & $\begin{array}{l}\text { L'Aigle Noir } \\
200 \mathrm{tx} \text {. }\end{array}$ & J. Pruncau & $\begin{array}{l}\text { J. Gitton } \\
\text { Fr. Duprat } \\
\text { A. Bouchel }\end{array}$ & & - & Québec & - - \\
\hline $\begin{array}{l}\text { B } 5678 \text { no } 347 \text {, } \\
1679,18 / 3 \text {. }\end{array}$ & $\begin{array}{l}\text { Le St Jean Bap- } \\
\text { tiste }\end{array}$ & P. Thomas & P. Gallon & & - & Id. & - - \\
\hline $\begin{array}{l}\text { B } 5678 \text { no } 349, \\
1679,10 / 4 .\end{array}$ & $\begin{array}{l}\text { Le M o u t o n } \\
\text { Blanc }\end{array}$ & J. de St Michel & - & & -- & Id. & - \\
\hline $\begin{array}{l}\text { B } 5678 \text { no } 350, \\
1679,7 / 5 \text {. }\end{array}$ & Le St Pierre & P. Bataillé & P. Gaigneur & & - & Canada & - - \\
\hline $\begin{array}{l}\text { B } 5678 \text { no } 353 \text {, } \\
\text { \& Teuleron }\end{array}$ & $\begin{array}{l}\text { Le St Agnes } \\
300 \text { tx. }\end{array}$ & J. Monbeuil & $\begin{array}{l}\text { J. Gitton } \\
\text { Fr. Duprat }\end{array}$ & $\begin{array}{l}\text { Les } \\
\text { res }\end{array}$ & propriétai- & Québec et pêche & - - \\
\hline $\begin{array}{l}1679,17 / 4 . \\
\text { B } 5678 \text { no } 355 \text {, } \\
1679,20 / 5 .\end{array}$ & Le St Joseph & N. Dalton & A. Boucnet & & - & - & - \\
\hline \multicolumn{8}{|c|}{1680} \\
\hline $\begin{array}{l}\text { B } 5679 \text { no } 377 \text {, } \\
1680,11 / 4 .\end{array}$ & $\begin{array}{l}\text { Le Perroquet } \\
100 \text { tx. }\end{array}$ & P. Montbeuil & $\begin{array}{l}\text { J. Gitton \& au- } \\
\text { tres }\end{array}$ & $\begin{array}{l}\text { Les } \\
\text { res }\end{array}$ & propriétai- & $\begin{array}{l}\text { Acadie, Québec } \\
\text { et pêche }\end{array}$ & - - \\
\hline $\begin{array}{l}\text { B } 5679 \text { no } 378 \text {, } \\
1680,17 / 4 .\end{array}$ & $\begin{array}{l}\text { Le M ou to n } \\
\text { Blanc, } 300 \text { tx. }\end{array}$ & A. Chaviteau & Cie du Canada & & - - & Québec & - - \\
\hline $\begin{array}{l}\text { B } 5679 \text { no } 380 \text {, } \\
1680,20 / 6 \text {. }\end{array}$ & $\begin{array}{l}\text { Le St Joseph, } \\
260 \text { tx. }\end{array}$ & N. Dalton & - & & - & Id. & -- \\
\hline $\begin{array}{l}\text { B } 5679 \text { no } 381 \text {, } \\
1680,22 / 6 \text {. }\end{array}$ & $\begin{array}{l}\text { Le St François } \\
\text { Xavier } 150 \text { tx. }\end{array}$ & J.-F. Bourdon & $\begin{array}{l}\text { Lebert de Mont- } \\
\text { royal }\end{array}$ & & - - & Id. & - \\
\hline $\begin{array}{l}\text { B } 5679 \text { no } 382 \text {, } \\
1680,7 / 2 \text { et } 22 / 12 \text {. }\end{array}$ & La Rochelaise & P. Gentet & - & & - & Plaisance & $\begin{array}{l}2 \text { roles, } 1 \text { en fóvrier, } \\
1 \text { en Décembre. }\end{array}$ \\
\hline $\begin{array}{l}\text { B } 5679 \text { no } 384 \text {, } \\
1680,14 / 2 .\end{array}$ & $\begin{array}{l}\text { La F or tune } \\
\text { Blanche }\end{array}$ & T. Chaillaud & A. Perez & & - & Id. & - \\
\hline
\end{tabular}


1681

\begin{tabular}{|c|c|c|c|c|c|c|}
\hline Source & Nom du navire & Maitre & Propriétaire & Armateur & Destination & Observations diverses \\
\hline $\begin{array}{l}\text { B } 5680 \text { no } 354 \text {, } \\
1681,23 / 5 .\end{array}$ & $\underset{\text { Blanc }}{\text { Le } M \text { ou to n }}$ & A. Chaviteau & - & - & Québec & - - \\
\hline $\begin{array}{l}\text { Teuleron } \\
1681,16 / 5\end{array}$ & $\begin{array}{l}\text { Le Patriarche } \\
\text { Jacob }\end{array}$ & - - & - & - - & - & - - \\
\hline Idem & La Catherine & - & - & - - & - - & - \\
\hline
\end{tabular}

1682

\begin{tabular}{|c|c|c|c|c|c|c|}
\hline $\begin{array}{l}\text { B } 5689 \text { no } 187 \text {, } \\
1682,28 / 1 .\end{array}$ & $\begin{array}{l}\text { La Catherine } \\
200 \mathrm{tx} .\end{array}$ & Hurtin & J. Raulé & - & $\begin{array}{l}\text { Canada et An- } \\
\text { tilles }\end{array}$ & - - \\
\hline $\begin{array}{l}\text { B } 5681 \text { no } 191 \text {, } \\
1682,3 / 3 \text {. }\end{array}$ & $\begin{array}{l}\text { La Providence } \\
\text { ex C } \mathrm{A} \text { t e } \mathrm{a} u \\
\text { d'Ambourg } 200 \\
\text { tx }\end{array}$ & J. Durand & A. Heron & - & Québec & - \\
\hline $\begin{array}{l}\text { B } 5681 \text { no } 193 \text {, } \\
1682,4 / 3 . \\
\text { B } 5681 \text { no } 194 \text {, } \\
1682,5 / 5 .\end{array}$ & $\begin{array}{l}\text { Le St Charles } \\
\text { La Ste Agnès }\end{array}$ & $\begin{array}{l}\text { P. Tharay } \\
\text { P. Montbeuil }\end{array}$ & Fr. Duprat & - & $\begin{array}{l}\text { Acadie } \\
\text { Québec }\end{array}$ & - \\
\hline $\begin{array}{l}\text { B } 5681 \text { no } 196 \text {, } \\
1682,9 / 5 \text {. }\end{array}$ & L'Honoré & P. Gravouil & - - & - - & Id. & \\
\hline $\begin{array}{l}\text { B } 5681 \text { no } 198, \\
1682,22 / 5 . \\
\text { B } 5681 \text { no } 200 \text {, } \\
1682,17 / 6 \text {. } \\
\text { B } 5681 \text { no } 201, \\
1682,8 / 7 \text {. }\end{array}$ & $\begin{array}{l}\text { Le St Jean Bap- } \\
\text { tiste } \\
\text { Le St François } \\
\text { Xavier } \\
\text { La Bretonne }\end{array}$ & $\begin{array}{l}\text { Ch. Chaviteau } \\
\text { J.-F. Bourdon } \\
\text { J. Borny }\end{array}$ & $\begin{array}{l}\text { P. Gallon et au- } \\
\text { tres. }\end{array}$ & - & $\begin{array}{l}\text { Id. } \\
\text { Id. }\end{array}$ & - \\
\hline
\end{tabular}




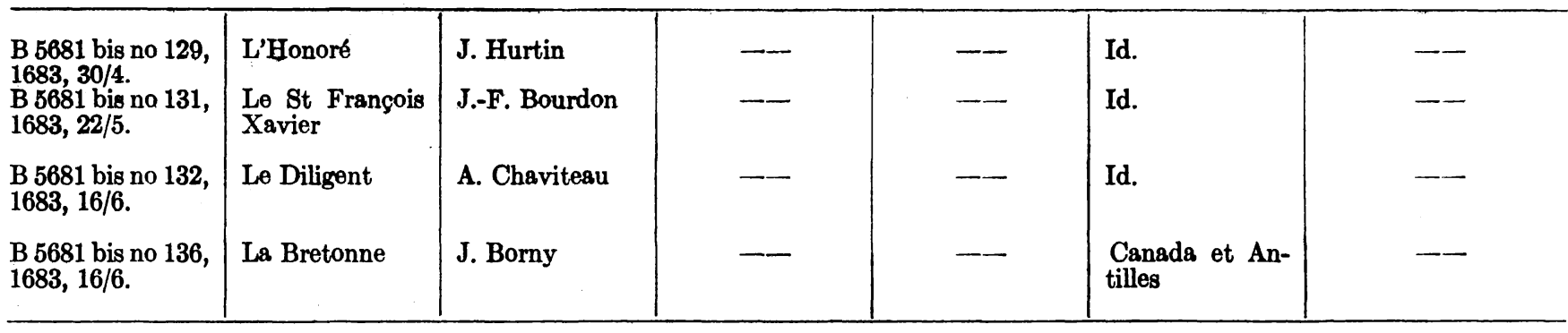

1684

\begin{tabular}{|c|c|c|c|c|c|c|}
\hline $\begin{array}{l}\text { B } 5682 \text { no } 367 \text {, } \\
1684,17 / 6 \text {. }\end{array}$ & $\begin{array}{l}\text { L'Honoré, } 200 \\
\text { tx. }\end{array}$ & J. Hurtin & J. Grignon & - & Canada et Iles & - \\
\hline $\begin{array}{l}\text { B } 5682 \text { no } 373 \text {, } \\
1684,2 / 7 \text {. et Rivi- } \\
\text { ere, } 1684,5 / 6\end{array}$ & L'Aimable & C. Aygron & J. Massiot & $\begin{array}{l}\text { Cavalier de la } \\
\text { Balle }\end{array}$ & Id. & \\
\hline $\begin{array}{l}\text { B } 5682 \text { no } 376, \\
1684,2 / 9 .\end{array}$ & $\begin{array}{l}\text { La Belle Made- } \\
\text { lon, } 70 \mathrm{tx} \text {. }\end{array}$ & M. Achard & L. Allaire & - & $\begin{array}{l}\text { Acadie et An- } \\
\text { tilles }\end{array}$ & -- \\
\hline $\begin{array}{l}\text { B } 5682 \text { no } 383 \text {, } \\
1684,4 / 9 .\end{array}$ & $\begin{array}{l}\text { La Renée-Marie } \\
\text { de St-Gilles }\end{array}$ & P. Gravouil & - & Cie du Canada & $\begin{array}{l}\text { Canada et An- } \\
\text { tilles }\end{array}$ & \\
\hline $\begin{array}{l}\text { B } 5682 \text { no } 384, \\
1684,3 / 3 \text {. }\end{array}$ & $\begin{array}{l}\text { La Ville de Lis- } \\
\text { bonne }\end{array}$ & P. Durand & Fr. Pachot & - & Id. & - \\
\hline $\begin{array}{l}\text { B } 5682 \text { no } 392, \\
1684,11 / 5 \text {. }\end{array}$ & Le St-Louis & M. Candé & Bergier & - & Acadie & - - \\
\hline $\begin{array}{l}\text { B } 5682 \text { no } 394, \\
1684,13 / 6\end{array}$ & $\begin{array}{l}\text { Le Samuel } 250 \\
\text { tx. }\end{array}$ & J. Duret & $\mathbf{A}$ et $\mathbf{S}$ Bernon & - & Canada & - \\
\hline $\begin{array}{l}\text { B } 5682 \text { no } 397 \text {, } \\
1684,8 / 7 \text {. }\end{array}$ & $\begin{array}{l}\text { Les Armes de } \\
\text { France }\end{array}$ & H. Abrard & - & - - & Id. & - \\
\hline
\end{tabular}


1685

\begin{tabular}{|c|c|c|c|c|c|c|}
\hline Source & Nom du navire & Maître & Propriétaire & Armateur & Destination & Observations diverses \\
\hline $\begin{array}{l}\text { B } 5683 \text { no } 296, \\
1685,31 / 3 .\end{array}$ & La Bretonne & J. Compere & - & $\begin{array}{l}\text { A. Heron pr les } \\
\text { Fermiers du Do- } \\
\text { maine d'Occi- } \\
\text { dent. }\end{array}$ & $\begin{array}{l}\text { Acadie et An- } \\
\text { tilles }\end{array}$ & - \\
\hline $\begin{array}{l}\text { B } 5683 \text { no } 304 \text {, } \\
1685,7 / 4 \text {. }\end{array}$ & L'Aigle Noir & J. Pruneau & Fr. Duprat & - & $\begin{array}{l}\text { Canada et An- } \\
\text { tilles }\end{array}$ & - - \\
\hline $\begin{array}{l}\text { B } 5683 \text { no } 309, \\
1685,23 / 5 .\end{array}$ & $\begin{array}{l}\text { La Diligente, } \\
250 \text { tx. }\end{array}$ & J. Durand & A. Heron & - & Id. & - \\
\hline $\begin{array}{l}\text { B } 5683 \text { no } 315 \text {, } \\
1685,26 / 5 .\end{array}$ & $\begin{array}{l}\text { La Vierge } 200 \\
\text { tx. }\end{array}$ & St Marcq & A. Bouchel & - - & $\begin{array}{l}\text { Acadie et An- } \\
\text { tilles }\end{array}$ & - - \\
\hline $\begin{array}{l}\text { B } 5683 \text { no } 321 \text {, } \\
1685,30 / 5 .\end{array}$ & La Marie 45 tx. & N. Despot & - & A. Heron & Id. & - \\
\hline $\begin{array}{l}\text { B } 5683 \text { no } 327 \text {, } \\
1685,30 / 5 \text {. }\end{array}$ & $\begin{array}{l}\text { Le Roi Salomon } \\
50 \text { tx. }\end{array}$ & J. Labrousse & - - & Id. & Id. & - \\
\hline $\begin{array}{l}\text { B } 5683 \text { no } 331 \text {, } \\
1685,30 / 6 .\end{array}$ & $\begin{array}{l}\text { Le St François } \\
\text { Xavier }\end{array}$ & J.-F. Bourdon & - - & - & Canada & \\
\hline $\begin{array}{l}\text { Rivière } \\
1685,13 / 4 .\end{array}$ & $\begin{array}{l}\text { La Marie Jo- } \\
\text { seph du Con- } \\
\text { quet, } 40 \text { tx. }\end{array}$ & J. Senne & - & - & $\begin{array}{l}\text { Acadie et An- } \\
\text { tilles }\end{array}$ & - \\
\hline
\end{tabular}




\begin{tabular}{|c|c|c|c|c|c|c|}
\hline $\begin{array}{l}\text { B } 5684 \text { no } 202, \\
1686,5 / 4 .\end{array}$ & $\begin{array}{l}\text { Le St François } \\
\text { Xavier }\end{array}$ & J.-F. Bourdon & - & - & Québec & - \\
\hline $\begin{array}{l}\text { B } 5684 \text { no } 203 \text {, } \\
1686,11 / 6 .\end{array}$ & $\begin{array}{l}\text { Le Nom de Jé- } \\
\text { sus }\end{array}$ & P. Durand & $\begin{array}{l}\text { Fr. Pachot \& } \\
\text { D. Busquet }\end{array}$ & - - & Id. & - - \\
\hline $\begin{array}{l}\text { B } 5684 \text { no } 209 \text {, } \\
1686,25 / 10 .\end{array}$ & L'Amitié 25 tx. & F. Flandreau & F. Flandreau & - - & Id. & - - \\
\hline $\begin{array}{l}\text { B } 5684 \text { no } 210, \\
1686,24 / 7\end{array}$ & $\begin{array}{l}\text { L'H o noré ou } \\
\text { Auguste Honoré }\end{array}$ & Z. Avrillou & J. Grignon & - - & $\begin{array}{l}\text { Canada et An- } \\
\text { tilles }\end{array}$ & - - \\
\hline $\begin{array}{l}\text { Rivière } \\
1686,15 / 6 .\end{array}$ & $\begin{array}{l}\text { La Marguerite } \\
\text { de Dieppe }\end{array}$ & D. Chrestien & - - & P. Garbuzat & - & - - \\
\hline $\begin{array}{l}\text { Rivière } \\
1686,10 / 8 .\end{array}$ & $\begin{array}{l}\text { La Diligente } \\
250 \text { tx. }\end{array}$ & J. Durand & $\begin{array}{l}\text { La Cie de pêche } \\
\text { d'Acadie }\end{array}$ & $\begin{array}{l}\text { Les propriétai- } \\
\text { res }\end{array}$ & $\begin{array}{l}\text { Chedabouctou } \\
\text { ret. a Marseille }\end{array}$ & - \\
\hline \multicolumn{7}{|c|}{1687} \\
\hline $\begin{array}{l}\text { B } 5685 \text { no } 138, \\
1687,30 / 5 .\end{array}$ & L'Union 120 tx. & Hurtin & A. Allaire & - & $\begin{array}{l}\text { Canada et An- } \\
\text { tilles }\end{array}$ & - \\
\hline $\begin{array}{l}\text { B } 5685 \text { no } 142 . \\
1687,12 / 5 .\end{array}$ & Le St François & F. Audiere & J. de Bortuste & - & Canada & - \\
\hline $\begin{array}{l}\text { B } 5685 \text { no } 147 \text {, } \\
1687,23 / 5 .\end{array}$ & $\begin{array}{l}\text { La Justice de } \\
\text { Calais, } 100 \text { tx. }\end{array}$ & A. Le Hoc & - & Fr. Pachot & Id. & - \\
\hline $\begin{array}{l}\text { B } 5685 \text { no } 152 \text {, } \\
1687,24 / 6 .\end{array}$ & La Diligente & J. Durand & frégate du Roi & - & Id. & - \\
\hline Rivière 1687, 5/3. & $\begin{array}{l}\text { Le Samuel } 200 \\
\text { tonneaux }\end{array}$ & J. Duret & $\begin{array}{l}\text { P. Faneuil \& } \\
\text { autres }\end{array}$ & $\begin{array}{l}\text { Ch. Aubert \& } \\
\text { autres }\end{array}$ & $\begin{array}{l}\text { Québec et An- } \\
\text { tilles }\end{array}$ & - \\
\hline $\begin{array}{l}\text { Rivière } \\
1687,14 / 2 .\end{array}$ & $\begin{array}{l}\text { La Bretonne, } \\
100 \mathrm{tx} .\end{array}$ & A. Ruelle & A. Heron & $\begin{array}{l}\text { Les propriétai- } \\
\text { res }\end{array}$ & Plaisance & - \\
\hline $\begin{array}{l}\text { Rivière } \\
1687,16 / 1 .\end{array}$ & $\begin{array}{l}\text { La Deprise } 100 \\
\text { tonneaux }\end{array}$ & Fr. Baudouin & Fr. Baudouin & R. Denys & $\begin{array}{l}\text { riviere St Lau- } \\
\text { rent }\end{array}$ & $\begin{array}{l}\text { pêche et port de mar- } \\
\text { chandises }\end{array}$ \\
\hline
\end{tabular}




\begin{tabular}{|c|c|c|c|c|c|c|}
\hline Source & Nom du navire & Maître & $\begin{array}{c}1688 \\
\text { Propriétaire }\end{array}$ & Armateur & Destination & Observations diverses \\
\hline $\begin{array}{l}\text { B } 235 \text { fos } 152 \& \\
\text { suiv. } 1688,23 / 3 \text {. }\end{array}$ & La:Maréchale & J. Guillot & R. Massiot & - & $\begin{array}{l}\text { Canada et An- } \\
\text { tilles }\end{array}$ & - \\
\hline $\begin{array}{l}\text { B } 235,1688,17 / 7 \\
\& \text { Riviere, } 1688 \text {, } \\
28 / 5 \text {. }\end{array}$ & $\begin{array}{l}\text { Le St Louis } 150 \\
\text { tx. }\end{array}$ & M. Cande & N. Claessen & Cie de l'Acadie & Acadie et pêche & - - \\
\hline B $235,1688,17 / 4$. & $\begin{array}{l}\text { LazSuzanne } 50 \\
\text { tx. }\end{array}$ & P. Duret & $\begin{array}{l}\text { P. Depont \& } \\
\text { consorts }\end{array}$ & - & Plaisance & - \\
\hline В $235,1688,21 / 4$. & $\begin{array}{l}\text { Le Soleil d'Afri- } \\
\text { que }\end{array}$ & M. de Corme & - & - - & Canada & - - \\
\hline B $235,1688,21 / 4$. & $\begin{array}{l}\text { Le Nom de Je- } \\
\text { sus }\end{array}$ & N. Blacquebot & - & - - & Québec & - \\
\hline В $235,1688,28 / 4$. & La Françoise & J. Pruneau & - & - & Québec & - - \\
\hline B $235,1688,28 / 4$. & $\begin{array}{l}\text { Le Dragon } 80 \\
\text { tx. }\end{array}$ & N. Noël & M. Villierme & - & $\begin{array}{l}\text { Canada et An- } \\
\text { tilles }\end{array}$ & - \\
\hline В $235,1688,31 / 6$. & Le St Honoré & A. Ruelle & Grignon & - & Canada & - \\
\hline B $235,1688,28 / 7$. & La Vierge & - & A. Bouchel & - & Id. & - - \\
\hline Riviere 1688 & $\begin{array}{l}\text { La Diligen te } \\
250 \text { tx. }\end{array}$ & J. Durand & - & Cie de l'Acadie & $\begin{array}{l}\text { Qúbbec, Cap au } \\
\text { charbon et An- } \\
\text { tilles }\end{array}$ & - \\
\hline
\end{tabular}




\begin{tabular}{|c|c|c|c|c|c|c|}
\hline $\begin{array}{l}\text { B } 235 \text {, fos } 186 \text { et } \\
\text { suiv. } 1689,21 / 2 \text {. }\end{array}$ & Le St Mathieu & M. Augizeau & - & - & $\begin{array}{l}\text { Bordeaux et Ca- } \\
\text { nada }\end{array}$ & - \\
\hline $\begin{array}{l}\text { B } 235,1689,23 / 4 \\
\text { et } 20 / 7 \text {. }\end{array}$ & $\begin{array}{l}\text { Le St François } \\
\text { Xavier }\end{array}$ & Fr. Pillet & - & - & Québec & - - \\
\hline $\begin{array}{l}\text { B } 235,1689,26 / 5 \\
\& \text { Riviere } 1689, \\
26 / 2 .\end{array}$ & Le St Jean & $\begin{array}{l}\text { le s. de Bona- } \\
\text { venture, capit. }\end{array}$ & 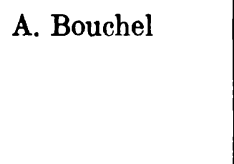 & $\begin{array}{l}\text { J. Gitton, } \\
\text { F. Duprat \& } \\
\text { Fr. Pachot, Drs } \\
\text { de la Cie de la } \\
\text { Baie du N. }\end{array}$ & Baie du Nord & - \\
\hline B $235,1689,13 / 7$. & L'Union & J. Chauvet & Cie d'Acadie & - & Acadie & - \\
\hline B 235, 1689, 20/7. & La Vierge & T. Metifeu & A. Bouchel & - & Canada & - \\
\hline \multicolumn{7}{|c|}{1690} \\
\hline $\begin{array}{l}\text { B 235, fos 206, \& } \\
\text { suiv. } 1690,23 / 3 \text {. }\end{array}$ & $\begin{array}{l}\text { Les Armes de la } \\
\text { Compagnie }\end{array}$ & $\begin{array}{l}\text { Le s. de Bona- } \\
\text { venture, cap. }\end{array}$ & - & - & $\begin{array}{l}\text { Canada et Mer } \\
\text { du Nord }\end{array}$ & - \\
\hline B $235,1690,1 / 4$. & L'Union & E. Rousseau & Cie de l'Acadie & - & Port Royal & - \\
\hline В $235,1690,15 / 6$ & La Vierge & T. Metifeu & A. Bouchel & - & Canada & - \\
\hline $\begin{array}{l}\text { B } 235,1690,29 / 7 \\
\& \text { Rivière } 1690, \\
7 / 7 \text {. }\end{array}$ & Le St Nicolas & E. Dolbecq & N. Claessen & $\begin{array}{l}\text { Le Commissaire } \\
\text { de la marine }\end{array}$ & Id. & - \\
\hline $\begin{array}{l}\text { B } 235,1690,29 / 7 \\
\& \text { Riviere } 1691 \text {, } \\
13 / 4 .\end{array}$ & $\begin{array}{l}\text { La Notre Dame } \\
\text { de Protection }\end{array}$ & N. Blacquebot & - & N. Grozé & $\begin{array}{l}\text { Acadie et An- } \\
\text { tilles }\end{array}$ & $\begin{array}{l}\text { Piis puis naufrage } \\
\text { devant Tadoussac en } \\
\text { Novembre }\end{array}$ \\
\hline $\begin{array}{l}\text { Rivière } \\
1690,13 / 4\end{array}$ & $\begin{array}{l}\text { Le St Francois } \\
\text { de Paule, } 120 \text { tx. }\end{array}$ & P. Durand & Fr. Pachot & Cie de Guince & $\begin{array}{l}\text { Canada et An- } \\
\text { tilles }\end{array}$ & - \\
\hline
\end{tabular}




\begin{tabular}{|c|c|c|c|c|c|c|}
\hline & & & 1691 & & & \\
\hline Source & Nom du navire & Maître & Propriétaire & Armateur & Destination & Observations diverses \\
\hline $\begin{array}{l}\text { B } 235 \text {, fos } 232 \& \\
\text { suiv. } 1691,2 / 5 \text {. }\end{array}$ & $\begin{array}{l}\text { La Famille de } \\
\text { Seudre, } 100 \text { tx. }\end{array}$ & J. Garesché & J. Groze & - & Canada & - \\
\hline B 235. & $\begin{array}{l}\text { Les Armes de la } \\
\text { Cie du Nord de } \\
\text { Canada }\end{array}$ & N. Robillard & - & - & Id. & - - \\
\hline B 235. & $\begin{array}{l}\text { Le Jean de Chail- } \\
\text { levette, } 80 \mathrm{tx} \text {. }\end{array}$ & J. Thomas & Descombes & - - & Id. & - \\
\hline В 235 . & $\begin{array}{l}\text { La Nouvelle } \\
\text { Paix, } 100 \mathrm{tx} .\end{array}$ & P. Montroux & $\begin{array}{l}\text { Bernonville, } \mathrm{Al}- \\
\text { laire \& Moreau }\end{array}$ & - & Canada & - - \\
\hline B 235 . & L'Aimable, $80 \mathrm{t}$. & J. Germon & $\begin{array}{l}\text { Bernonville \& } \\
\text { Faneuil }\end{array}$ & - & Id. & - \\
\hline B 235, 30/4. & $\begin{array}{l}\text { Le St François } \\
\text { de Paule }\end{array}$ & M. Gravouil & - - & - - & Id. & - \\
\hline B 235. & Le St Mathieu & S. Benesteau & - & - & Id. & - \\
\hline B 235, 5/7. & Le St Jean & J. Chauvet & A. Bouchel & - & - - & - \\
\hline B 235 . & $\begin{array}{l}\text { La Fleur de Mai } \\
\text { de Chaillevette }\end{array}$ & J. Morgat & - & - & - & - \\
\hline B 235. & Le St Nicolas & Dolbecq & N. Claessen & - - & $\begin{array}{l}\text { Canada et An- } \\
\text { tilles }\end{array}$ & - \\
\hline B 235, 7/5. & $\begin{array}{l}\text { La Notre Dame } \\
\text { du Rosaire }\end{array}$ & M. Congerie & - & -- & - & - - \\
\hline B $235,10 / 7$. & L'Honoré & J. Rateau & - & - & - & - \\
\hline
\end{tabular}




\begin{tabular}{|c|c|c|c|c|c|c|c|}
\hline $\begin{array}{l}\text { B } 235, \text { fos } 253, \& \\
\text { suiv. } 1692,2 / 5 \text {. }\end{array}$ & Le St Joseph & J. Couillandeau & - & & - & Québec & - \\
\hline B 235, 2/5. & La Ste Anne & J. Lagrange & - - & & - & Canada & - - \\
\hline B 235, Mai & Les Armes de la & Ch. François & - & & - & Québec & - - \\
\hline B 235, Mai & $\begin{array}{l}\text { Le Neptune, } \\
150 \text { tx. }\end{array}$ & P. Chevalier & $\begin{array}{l}\text { A. Mouchard } \\
\text { F. Pachot }\end{array}$ & & - - & Canada & - - \\
\hline B 235, Juin & La ville de Bor- & J. Delalande & H. Grozé & & - & Québec & - \\
\hline B 235, Juin & La Providence & J. Tesseron & Id. & & - - & & - \\
\hline B $235,10 / 5$. & Le St Honoré & J. Rateau & - & & - & Canada et Iles & - \\
\hline $\begin{array}{l}\text { Riviere } 1692,11 / 3 \\
\text { et } 2 / 6 \text {. }\end{array}$ & $\begin{array}{l}\mathrm{L} \text { e S t J a c o b } \\
\text { d'Altona, } 250 \text { tx }\end{array}$ & C. Eckhof & - & J. Git & itton & $\begin{array}{l}\text { Bordeaux, Que- } \\
\text { bec, Le Cap au } \\
\text { charbon \& An- } \\
\text { tilles. }\end{array}$ & - \\
\hline \multicolumn{8}{|c|}{1693} \\
\hline $\begin{array}{l}\text { B } 235 \text {, fo } 267 \text { \& } \\
\text { suiv. et Rivière } \\
1693,26 / 3 \text {. }\end{array}$ & $\begin{array}{l}\text { La Reine Marie, } \\
120 \text { tx. }\end{array}$ & J. Germon & $\begin{array}{l}\text { Bernon et Fa- } \\
\text { neuil J. Gitton } \\
\text { Brouiliant Gr }\end{array}$ & $\begin{array}{l}\text { Les } \\
\text { res }\end{array}$ & propriétai- & Plaisance & Pêche et troque. \\
\hline $\begin{array}{l}\text { B } 235, \text { Avril. Ri- } \\
\text { vière } 1693,29 / 4 \text {. }\end{array}$ & $\begin{array}{l}\text { Le St François } \\
\text { de Paule }\end{array}$ & J. Duhulque & $\begin{array}{l}\text { A. Mouchard } \\
\text { P. Depont }\end{array}$ & $\begin{array}{l}\text { Les } \\
\text { res }\end{array}$ & propriétai- & Plaisance & Pêche et troque. \\
\hline B 235, Avril. & Le Pontchar- & J. Javelleau & & & - - & Canada & - - \\
\hline B 235, Avril. & $\begin{array}{l}\text { Le St Louis de } \\
\text { Québec }\end{array}$ & $\begin{array}{l}\text { N. Esgron La- } \\
\text { mothe }\end{array}$ & - - & & - - & Id. & - - \\
\hline $\begin{array}{l}\text { B } 235 \text { \& Rivière, } \\
1693,29 / 4 \text {. }\end{array}$ & $\begin{array}{l}\text { La Fille bien ai- } \\
\text { mé, } 250 \text { tx. }\end{array}$ & J. Duret & $\begin{array}{l}\text { I. Mouchard \& } \\
\text { J. Gitton }\end{array}$ & $\begin{array}{l}\text { Les } \\
\text { res }\end{array}$ & propriétai- & $\begin{array}{l}\text { Québec et Plai- } \\
\text { sance }\end{array}$ & - \\
\hline B 235. & Le St Joseph & J. Couillandeau & - & & - - & Canada & - - \\
\hline Rivière $1693,29 / 4$. & La Perle & J. Vivien & P. Garbuzat & & - - & Québec & - - \\
\hline
\end{tabular}

